\title{
1 HieRFIT: Hierarchical Random Forest for Information Transfer
}

2 Yasin Kaymaz ${ }^{1}$, Florian Ganglberger ${ }^{2}$, Ming Tang ${ }^{1}$, Francesc Fernandez-Albert ${ }^{3}$, Nathan

3 Lawless $^{3}$, Timothy Sackton ${ }^{1}$

$4 \quad{ }^{1}$ Informatics Group, Harvard University, Cambridge, MA, USA.

$5 \quad 2$ VRVis Research Center, Vienna, Austria.

$6{ }^{3}$ Global Computational Biology \& Digital Sciences, Boehringer Ingelheim Pharma GmbH \& Co KG, 7 Biberach an der Riss, DE. 


\section{Abstract}

The emergence of single-cell RNA sequencing (scRNA-seq) has led to an explosion in novel

10 methods to study biological variation among individual cells, and to classify cells into functional and

11 biologically meaningful categories. Here, we present a new cell type projection tool, HieRFIT

12 (

13 HieRFIT uses a priori information about cell type relationships to improve classification accuracy,

14 taking as input a hierarchical tree structure representing the class relationships, along with the

15 reference data. We use an ensemble approach combining multiple random forest models, organized

16 in a hierarchical decision tree structure. We show that our hierarchical classification approach

17 improves accuracy and reduces incorrect predictions especially for inter-dataset tasks which reflect

18 real life applications. We use a scoring scheme that adjusts probability distributions for candidate

19 class labels and resolves uncertainties while avoiding the assignment of cells to incorrect types by

20 labeling cells at internal nodes of the hierarchy when necessary. Using HieRFIT, we re-analyzed

21 publicly available scRNA-seq datasets showing its effectiveness in cell type cross-projections with

22 inter/intra-species examples. HieRFIT is implemented as an R package and it is available at

\section{3 (https://github.com/yasinkaymaz/HieRFIT/releases/tag/v1.0.0)}

25 Corresponding Author: Timothy Sackton 


\section{Introduction}

Single-cell RNA-seq (scRNA-seq) technology has provided an unparalleled picture of the

28 cell-to-cell complexity of biology in multicellular organisms. As technological improvements have

29 allowed increasingly large studies, comprehensive cell atlas experiments have revealed

30 unprecedented cell-to-cell heterogeneity and molecular dynamism of cell types across both human

31 and model organisms (Cao et al., 2017, Rosenberg et al., 2018). Single-cell genomics have enabled

32 tracing developmental lineages of early embryonic cells and building transcriptional landscapes of

33 organogenesis at single-cell resolution, and uncovering novel rare cell populations (Cao et al., 2019,

34 Tabula Muris et al., 2018).

35 As single-cell experiments grow in size and scope, the computational challenges associated

36 with analyzing and interpreting these data are also growing. In particular, identifying cell types

37 present in a sequenced population is critically important for enabling biological insight. Widely

38 prevalent single cell analyses protocols incorporate unsupervised clustering methods as a key step

39 in this process. For example, k-means, hierarchical clustering, KNN (k-nearest neighbor) or SNN

40 (shared-nearest-neighbor) graphs, and Louvain community detection are all used in a variety of

41 different packages, such as SC3 (Kiselev et al., 2017) and Seurat (Butler et al., 2018). Unsupervised

42 clustering methods attempt to identify a consistent and biologically meaningful set of cell types or

43 cell states in an experiment, usually via a projection of high dimensional data. These approaches

44 have identified numerous novel subtypes (Aevermann et al., 2018, Plasschaert et al., 2018, Suo et

45 al., 2018), although complexities of parameter optimization, number of available cells, and intrinsic

46 noise of single-cell data can pose challenges (Kiselev et al., 2019, Tang et al., 2020).

47 However, unsupervised clustering approaches do not provide any rapid or automated way

48 of defining cluster identities, which is often done by manually checking marker gene expression. In

49 addition to being cumbersome, manual annotation depends on the robustness of a handful of a

50 priori marker genes. When cell types are highly similar to each other transcriptomically, manual 
51 annotation may be prone to human error as reliable and obvious marker genes may not exist

52 (Lähnemann et al., 2020). An alternative to unsupervised clustering is to use the rich information

53 from larger atlas projects, and focus on information transfer to new studies (Wilbrey-Clark et al.,

54 2020). While potentially faster and more accurate for cell type annotation than unsupervised

55 clustering, especially for small-scale studies, integration and accurate information transfer between

56 existing atlas datasets play a critical role. Supervised machine learning methods, using large cell

57 atlas datasets as training data, provide a potential approach to automate information transfer for

58 faster and accurate projections (Petegrosso et al., 2020).

A number of supervised classification methods have been developed, including singleCellNet (Tan and Cahan, 2019), ACTINN (Ma and Pellegrini, 2019), Garnett (Pliner et al., 2019), with different strengths and limitations. These methods differ in various aspects such as

62 feature selection, for instance, singleCellNet trains its models with random forest after extracting a

63 set of feature pairs from the reference data while ACTINN uses neural networks that automatically

64 chooses the features. Garnett, on the other hand, relies only on a set of cell type specific marker

65 genes as input independent of a reference dataset. Although the majority of these developed

66 methods are flat classifiers, hierarchical classification has also been implemented in the single-cell

67 context with CHETAH (de Kanter et al., 2019), and scClassify (Lin et al., 2019), which allowed

68 intermediate class assignments, although their outputs provided limited insight into actual cell

69 types.

Despite the rapid proliferation of cell-type assignment methods, a number of limitations still

71 exist with current approaches. Many existing methods work best when the reference training data

72 is composed of a few well-represented cell types, and when the query data contains a few or no

73 novel types (Abdelaal et al., 2019a). However, an ideal classification should be able to handle many

74 candidate cell classes, potentially hundreds, and not rely on a minimum input threshold of query 
75 data, as some single-cell protocols produce low-throughput data in which rare cell types are

76 represented with only a few cells (Campbell et al., 2017). In addition, handling complex

77 classification tasks by conventional methods usually involves either assigning to a cell type with

78 low confidence or the best case is declaring them as 'undetermined'. However, this approach

79 underestimates the potentially informative biological signal which is often challenging to harvest

80 and valuable to resolve experimental questions. Furthermore, considering cell types as discrete

81 entities with clear boundaries is far from ideal as, in reality, many cells are in transitioning

82 intermediate stages, which makes classification more compelling (Macaulay et al., 2016). Thus,

83 alternative approaches that benefit from hierarchical consideration of cell types are required to

84 eliminate these issues in the single-cell identity detection.

Here, we propose a new hierarchical classification approach, HieRFIT, which uses a

86 hierarchical tree structure of reference cell clusters, allowing custom defined intermediate classes

87 (internal nodes) that have biological meaning. Using this hierarchical model, we both improve

88 HieRFIT's ability to provide accurate cell type classification, and allow cells that cannot be

89 accurately classified to be assigned to the best supported internal node. We implemented our

90 approach as an R package and tested against various classification tasks. 


\section{Methods}

\section{Constructing the cell type decision tree with class hierarchies}

A key input component of HieRFIT is the cell type hierarchical tree. We define this hierarchy

94 as a tree, $\tau$, which is a subtype of directed acyclic graphs, where each cell type or cell class is

95 represented as a node $v$, and the connections between the nodes are edges, E. Nodes can only have

96 a single parent, but can have multiple child nodes. The nodes in the tree, $\tau$, are also asymmetric

97 (each child node cannot be a parent of its own parent), and the tree itself is transitive (each node is

98 also a child node of its parent's ancestral node). From this cell type tree, we can define an ancestral

99 hierarchy. Let $A$ represent a set of all ancestral nodes of a given node and $Y$ represent the set of

100 class labels of all nodes. Then, $A_{j}=\left\{v_{j}, v_{j+1}, v_{j+2}, \ldots, v_{k}\right\}$ is the set of nodes comprising the ancestral

101 path for node $v_{j}$ reaching up to the root node $v_{k}$ and $Y_{j}=\left\{y_{1}, y_{2}, \ldots, y_{n}\right\}$ is the class label set for

102 children of node $v_{j}$. We define the terminal nodes with no children as leaves. To define this

103 hierarchical tree for a given reference datasets, the user can input a cell type table in a tab delimited

104 format with each row designates a leaf cell type from the reference dataset and columns represent

105 the intermediate cell types to be used as internal nodes (Supplementary Table 1). HieRFIT can

106 also create a de novo tree out of cell type distances based on their averaged gene expressions using

107 hierarchical clustering if an input tree is not provided.

\section{Feature selection from reference data and local classifier training}

Feature selection is performed for each local classifier (internal node) separately. Let $M$ be

110 the normalized expression matrix to be used as a training data, which is composed of genes $G$ and

111 samples (cells) $X$ accompanied by a set of class types $Y$. In addition to the existing class types, an

112 'OutGroup' class that represents the other cell types is also added to the set, $Y$. 'OutGroup' class

113 sample size is limited to a maximum 500 cells (same as other classes, and can be altered by user) 
114 for all nodes and is formed by randomly selecting cells from classes that are not present for the

115 local classifier. HieRFIT selects a set of separate features, $f_{j} \in G$, for every internal node, $v_{j} \in v$,

116 using the corresponding subset data $m_{j} \subset M$. Genes with very limited variation across cell types, $m_{j}$

$117\left(\sigma^{2}<0.01\right)$, are pre-filtered in order to eliminate non-informative features. After standardizing the

118 expression by centering at the mean and scaling by the standard deviation, HieRFIT computes

119 eigenvectors of the data with principal component analysis using the 'prcomp' function from the $\mathrm{R}$

120 stats package. To define features, HieRFIT first selects principal components (PCs) that usefully

121 separate class labels $Y_{j}$, by computing a t-test on the component scores of each PC and selecting PCs

122 with $P<0.05$ (following Bonferroni correction). To turn these informative PCs into highly variable

123 feature sets, HieRFIT chooses the top 2000 variables (genes) that are most correlated with their

124 eigen vectors based on absolute component loading values (number of top genes selected can be

125 changed by user). The number of genes selected from each component is proportional to variance

126 explained by the PC. Further, wilcoxon rank sum test between the class labels is applied to further

127 select (by default) 200 differentially expressed genes (based on adjusted p-values) as features to be

128 used in local classifier training.

HieRFIT constructs a reference classifier with multiple local classifiers, one for each of the

130 internal nodes on the hierarchical tree. Local classifiers are created using a random forest algorithm

131 implemented with the R package Caret, with the features selected separately for each node using

132 the procedure described above, and with 500 trees (by default). The training sample set of each

133 local classifier, $m_{j}$, corresponds to the cells from reference data with cell type labels matching the

134 class labels of the node's children, $Y_{j}$. The array of local classifiers is stored as an S4 object (in R) in

135 the hierarchical organization to be used for projecting cell types on a query data. 


\section{Sigmoid calibration and noise injection}

The random forest classifier produces as output a vector of votes for each class, one from

139 each tree. However, these vote distributions are not equivalent to class probabilities, therefore,

140 they need to be transformed with a calibration function before they can be used as probabilities.

141 HieRFIT implements Platt scaling (Platt, 1999) for this purpose: as the final step of creating a

142 HieRFIT model, we construct a sigmoid function on reference data with class labels using a

143 multinomial logistic regression implemented in the 'nnet' R package. This sigmoid function allows

144 the conversion of class votes as the unprocessed output of random forest classifier to class

145 probabilities. In order to provide a certain level of flexibility against dropout events in scRNA-seq,

146 we also implemented a noise injection step prior to generating the sigmoid function (Zur et al.,

147 2009). Noise injection occurs by setting expression values of a subset of randomly selected feature

148 sets of each local classifier (by default $10 \%$ of all features) to zero.

\section{Asymmetric entropy-based certainty measurement}

In order to convert class probabilities into class assignments, allowing for the possibility

151 that some cells cannot be accurately assigned, we implemented a certainty function per candidate

152 class. We used asymmetric entropy measurement (Marcellin et al., 2006) in our certainty function

153 as follows;

Let $p_{i}$ denote probability of class $y_{i} \in Y_{j}$ at the node $v_{j}$, then, the asymmetric entropy as a

155 measure of uncertainty is

$$
h\left(p_{i}\right)=\frac{p_{i}\left(1-p_{i}\right)}{\left(1-2 w_{i}\right) p_{i}+w_{i}^{2}}
$$

157 where $w_{i}$ is probability of $y_{i}$ at which maximum uncertainty is achieved. Note that $h$ is equal to 158 quadratic entropy of Gini when $w_{i}=0.5$ in binary class modalities. From $h$, we then derived a 
159 function called 'certainty function', $U$, which contains an additional coefficient, $\lambda$, to assign

160 directionality as follows;

$$
U=\lambda \cdot\left[1-h\left(p_{i}\right)\right] \text { where } \begin{cases}\lambda=1 & \text { if } p_{i} \geq w_{i} \\ \lambda=-1 & \text { if } p_{i}<w_{i}\end{cases}
$$

162 Certainty scores center at zero when $p_{i}=w_{i}$ and range between -1 and 1 representing maximum

163 certainties about unrelatedness and relatedness to the class, respectively. In order to obtain a set of

164 empirical probability centroids, $W=\left\{w_{1}, w_{2}, \ldots, w_{i}, \ldots, w_{n}\right\}$, for each class, HieRFIT randomizes the

165 feature set $f_{j}$ of $m_{j} \subset M$ for the corresponding node with random permutations and calculates

166 expected probabilities of each class as the mean across iterations.

\section{Scoring scheme and decision rule for class projection}

HieRFIT then scores each in a "top-down" manner, which refers to taking all ancestral node scores and their metrics into account beginning from root node. In order to project class labels from the HieRFIT model created using the reference dataset and a cell type tree, the first step is to obtain

171 an array of certainty scores from all local classifiers for all class types. Let $x \in X$ be a cell in a query

172 dataset. In order to determine class type of $x$, HieRFIT generates an array of path certainty scores

$173 U_{i j}(x)$ which is calculated using classification certainty scores for every node on the ancestral path,

174 where $i$ represents the class types of node $j$ by traversing the tree and following the ancestral path

175 reaching to the $\operatorname{root} v_{k}$ as follows;

$$
U_{i j}(x)=\sum_{v_{j} \in A}^{k} U_{j}(x)-U_{j}^{\text {sib,out }}(x)
$$

177 where $U_{i j}(x)$ is the path certainty score of cell $x \in X$ for class $i$ in the node $v_{j}$, and $U_{j}^{\text {sib,out }}$ is the 178 sum of all siblings and outgroup certainty scores for the classifier node $v_{j}$.

179 During the score aggregation for decision, our rule for assigning class labels is 


$$
\operatorname{Class}(x)= \begin{cases}\underset{y_{i} \in Y}{\operatorname{argmax}} U_{i j}(x) & \text { if } U_{i j}(x)>\alpha \\ { }^{\prime} \text { Undetermined' } & \text { if } U_{x}=\emptyset\end{cases}
$$

181 HieRFIT assigns the maximum scoring class label out of all candidate classes that pass a certainty

182 threshold, $\alpha$. If none of the nodes passes, "Undetermined" is returned as a class label. The certainty

183 threshold is set to 0.05 by default, but can be changed by the user.

\section{Performance evaluations}

For intra-dataset performance evaluation tasks, we used 5-fold cross validation, in other words, training models with $80 \%$ and testing them on $20 \%$ of data. As the evaluation metric, we

187 calculated precision, recall, and F-measure averaged across iterations of cross validation. For interdataset evaluation tasks, in which training and test data originate from two separate datasets, we relied on the concordance between prior and predicted cell types of query datasets. We excluded

190 intermediate cell type, 'undetermined', and multi-class assignments in metric calculations. In

191 addition, given that HieRFIT uses a non-mandatory leaf node prediction approach and can return

192 intermediate class labels, we also accounted for intermediate cell type assignments in performance

193 evaluation. Therefore, we utilized precision, recall, and F-measure calculations modified for

194 hierarchical classifications (Kiritchenko et al., 2005). For these, let $A_{y}$ be a set of all ancestral labels

195 for the predicted class label $y$ and $A_{\theta}$ be the set of all ancestral labels for the true class $\theta_{x}$ of test

196 sample $x$, then hierarchical precision $(h P)$ and recall $(h R)$ are

$$
h P=\frac{\sum_{x \in X}\left|A_{y} \cap A_{\theta}\right|}{\sum_{x \in X}\left|A_{y}\right|} \quad h R=\frac{\sum_{x \in X}\left|A_{y} \cap A_{\theta}\right|}{\sum_{x \in X}\left|A_{\theta}\right|}
$$

198 where $\left|A_{v} \cap A_{\theta}\right|$ is the number of intersecting nodes between ancestors of predicted and true class

199 labels. Then, the hierarchical F-measure $(h F)$ is

$$
h F_{\beta}=\frac{\left(\beta^{2}+1\right) \cdot h P \cdot h R}{\beta^{2} \cdot h P+h R}, \quad \text { where } \beta=1
$$




\section{Datasets}

We analyzed PBMC scRNA-seq data from 10X Genomics with 2,700 single-cells by following

203 standard processing workflow as instructed on Seurat online tutorials

204 (https://satijalab.org/seurat/v3.1/pbmc3k_tutorial.html) (Butler et al., 2018). The main steps of

205 this analysis were data quality control and normalization, identifying variable genes, data scaling,

206 dimension reduction, clustering, finding differentially expressed genes, and assigning cell type

207 identities to clusters based on known cell markers. We used another public PBMC scRNA-seq

208 dataset with $68 \mathrm{~K}$ cells (Zheng $68 \mathrm{~K}$ ) as one of the reference datasets to generate a HieRFIT model

209 (Zheng et al., 2017b). We followed the same analysis steps in the publication (and code in GitHub

210 https://github.com/10XGenomics/single-cell-3prime-paper). We relabeled the cell types for easier

211 interpretation.

212 To evaluate the prediction performances with various data types, we selected several

213 published single-cell datasets with available class types and expression data (Supplemental Table

214 2). We used the same cross validation folds of these datasets previously generated for

215 benchmarking and performance evaluations through intra and inter-dataset challenges (Abdelaal et

216 al., 2019b). These datasets originate from 10 separate scRNA-seq studies, some with multiple levels

217 of cell type annotations. 


\section{Results}

\section{Overview of the algorithm}

We approached the cell type classification task as a hierarchical decision problem with a set

221 of predefined class relations. Our assumption of relationship between sub-classes and upper level

222 classes does not necessarily have to reflect biologically defined developmental trajectories but

223 rather represents organization of broader categories for cell type identities. HieRFIT uses multiple

224 local random forest classifiers, organized in a higher-level hierarchical decision tree, to split the

225 complex tasks into smaller and simpler ones. Here, we give a brief overview of the method, which is

226 described more in details in the Methods. Given a reference expression matrix, HieRFIT first

227 extracts the most informative principle components (PCs), which distinguish reference class types.

228 Then, it selects a set of genes from those components as predictors based on their correlations with

229 eigenvectors. Using the predictor set, it trains one classifier with corresponding subset data for

230 each parent node on a user defined hierarchical tree and builds a reference classifier. In order to

231 accurately project information from reference dataset on new experiments, we also implemented a

232 scoring scheme for assigning class labels in a non-mandatory leaf node prediction manner, which

233 allows us to provide intermediate cell types with broader context when data fails to provide enough

234 resolution for more specific cell types. The uncertainty function that we derived utilizes the

235 empirically learned background probability distribution and helps to determine whether observed

236 probability is informative for inferring class types. Our certainty based scoring scheme properly

237 finds the most likely ancestral path on the hierarchical cell type tree, which also provides additional 238 confidence about identity of query cells. 


\section{Hierarchical model construction and its algorithm}

243 corresponding reference cell types as prior information. The hierarchical tree of cell type can be

244 customized by the user with proper intermediate types. If the user lacks such prior information,

245 HieRFIT can generate the hierarchy de novo by computing the distances between the reference cell

246 types based on mean transcriptome expressions. To construct the reference HieRFIT model

247 (HierMod), we implement a six step protocol that is repeated for every internal node on the

248 hierarchical tree (Figure 1A). For each node a local classifier is generated using a random forest

249 classification algorithm. To prepare the reference expression data for model training, the first step

250 is to extract a cell data matrix that corresponds to the node. Training data is relabeled by bundling

251 the grandchildren under the node's children labels and adding an outgroup class as the representer

252 of other classes. A principal component analysis using the relabeled data provides the components

253 that are highly variable across cells and we select the components whose loadings significantly

254 separates cells with shared type from the others. The selected significant components allow us to

255 reduce the total number of genes to a highly variable gene set among which we select the final

256 feature set following the Wilcoxon rank sum test. This final feature set is used in the training of the

257 local classifier with the cell type labels (or relabels). All local classifiers are stored as an array of

258 models which are organized in accordance with the input tree hierarchy.

\section{Path certainty score computation and class selection}

Classical measures used in decision trees such as Shannon's entropy or quadratic entropy of

261 Gini are not suited well for real life imbalanced data with their symmetry assumptions for

262 equiprobability distributions among classes (Zighed et al., 2010). Therefore, we created an

263 alternative certainty function derived from asymmetric entropy (see methods). The main stage of

264 assigning a reference cell type to a query cell is to compute the array of certainty (U) values for each 
265 internal and terminal node (Figure 1B). In order to obtain such a metric, HieRFIT takes the gene

266 expression array of the query cell and the reference model (HierMod) as inputs. Along with the

267 gene expression array of the query cell, a randomized (shuffled 1000 times) expression array for

268 the same query is generated. Class votes from local classifiers are obtained for both observed and

269 shuffled expression arrays, simultaneously. Normalized class votes gathered from the local

270 classifiers are converted to class probabilities with logistic regression (aka sigmoid calibration)

271 function. The observed expression array is used to acquire adjusted class probabilities from each

272 local classifier, while the shuffled expression array is used to determine class centroids. These

273 centroids are used as certainties of the classes for the query with the observed class probabilities

274 using the certainty function (see Methods for details).

275 After computing the certainty values of each node for the query, we calculate the path

276 certainty scores for all candidate nodes by adding up the certainty values along the ancestral path

277 and subtracting all outgroup and sibling nodes certainties (Figure 1C). This path score defines the

278 final value for the nodes to be considered as candidate classes. The decision stage simply consisted

279 of choosing the maximum scoring node among the ones whose score exceeds a certain threshold

280 (alpha). In cases where no class exceeds the threshold, HieRFIT returns an "Undetermined". This

281 decision scheme permits internal nodes to be cell types of the query as well as leaf nodes that are

282 constrained with input the reference data in the first place.

\section{PBMC cell type classification with HieRFIT}

For demonstration purposes, we generated a reference model using the 68K PBMC single-

285 cell dataset from Zheng et al (Zheng et al., 2017b). We created an example hierarchical tree which

286 organized the reference cell types into two main groups, myeloid lineage and lymphoid lineage,

287 along with the hematopoietic stem cells (HSC) using the input tree file in Supplemental Table 1

288 (Figure 2A). Two main groups branched into further intermediate groups and general cell types. 
The terminal nodes comprise reference cell types from the PBMC data. We used this custom made hierarchical tree to create a HieRFIT model with maximum 500 cells per cell type in the training

291 process.

To test our hypothesis that our hierarchical classification approach provides more accurate and meaningful results as compared to conventional way of cell type identification, we used a toy dataset, another 3K PBMC, as the query (10X Genomics, https://support.10xgenomics.com/singlecell-gene-expression/datasets/1.1.0/pbmc3k). This publicly available dataset has been generated by $10 \mathrm{X}$ Genomics and processed through the Seurat pipeline. We followed the same guideline as in the Seurat tutorial to identify cell types with no prior information. This manual type annotation involves several commonly accepted processes, such as finding the variable genes, dimension reduction, and clustering of cells. Finally, determining the cell types involves manually checking the marker gene expression that are differentially expressed in clusters against the rest of the groups.

301 This process results annotation of 3K PBMC data with cell types: 'B cells', 'Megakaryocytes', 'CD14+ 302 Monocytes,' ‘CD16+ Monocytes', ‘Dendritic cells', ‘NK cells', ‘CD8 T cells', ‘CD4 T Naïve’ and 'Memory 303 cells' (Figure 2B, left). Then, we tested the hierMod we created with the 68K PBMC data by

304 projecting the reference cell types on the same 3K PBMC dataset. HieRFIT projections labeled the 305 cell in the query with leaf node labels as well as intermediate cell type defined in the model hierarchical tree above (Figure 2B, right). HieRFIT projections demonstrated a significant

307 concordance with Seurat cell types for the distinct cell types, such as 'B cells', 'Megakaryocytes', 'NK 308 cells', and 'Dendritic cells' (although these cells' HieRFIT projections were "Classical DCs" rather 309 than the parent node "Dendritic cells" on the tree). Seurat 'CD8 T cells' were labeled extensively 310 with subtypes "CD8 T GZMK+" and "CD8 T Cytotoxic" as well as their parent node "CD8 T cells", 311 partially (Figure 2C). "CD4 T Memory" cells were labeled mainly as "CD4 T Memory" and "CD4 T

312 Reg" in addition to the parent node "CD4 T cells". "CD4 T Naive" cell group, on the other hand, 313 received labels from almost all CD4 T sub-levels cell types and intermediate types such as “CD4 T 
314 cells" or even "T cells" as higher nodes on the tree. Interestingly, a group of cells within naïve CD4 T

315 cells received CD8 T cell labels, especially “CD8 T Naïve Cytotoxic”. Similarly, a subgroup of “CD14+

316 Monocytes" were labeled as “CD16+ Monocytes”, while all “CD16+ Monocytes” were correctly

317 labeled by HieRFIT. A small group of cells from the CD14+ cells were labeled as a parent node

318 "Monocytes".

\section{HieRFIT classifications are concordant with marker gene expressions}

To investigate the discordance between Seurat annotations and HieRFIT projections for

321 some of the cell groups, we further explored the marker gene expressions and their distribution. As

322 the heatmap of the confusion matrix demonstrates, 7 out of 9 cell types were labeled with cell types

323 by HieRFIT with more than $80 \%$ concordance (Figure 3A). Two of the cell types, "CD14+

324 Monocytes" and "CD4 T Naïve" received classification labels that resulted in $67.7 \%$ and $73.5 \%$

325 concordance, respectively. For the "CD4 T Naïve" cell types, we examined the expression

326 distribution of the CD8 T cell markers, CD8A and CD8B, as well as major CD4 markers for naïve

327 cells, IL7R and CCD7 (Figure 3B).

Within this group, the subset of cells which are predicted by HieRFIT to be CD8 T cells or its

329 subtypes expressed CD8A and CD8B at high levels, suggesting these cells are properly assigned to

330 the CD8 subtype (Figure 3B, upper panel - violin plots). The co-expression of the two markers also

331 clearly showed that the significant majority of these cells in fact expressed at least one of these

332 markers or both at the same time (Figure 3B, upper panel - UMAP panel with co-expression

333 projections). This observation supports the accuracy of HieRFIT projections that, in fact, these cells

334 are a class of CD8 T cells rather than CD4 T cells. On the other hand, the group of cells that were

335 concordantly labeled as CD4 T cells or its subtypes carried the proper CD4 T naïve marker

336 expressions, IL7R and CCR7, in line with their projected cell types (Figure 3B, lower panel - violin

337 plots and UMAP projections). 
We also investigated another group of cells with discordant predictions in "CD14+

Monocytes". Of these monocytes, 27.7\% were predicted as "CD16+ Monocytes". When we examined

the marker gene expression in cells classified as "CD14+ Monocytes" by Seurat, we observed that a significant portion of them expressed CD16 (FCGR3A) monocyte marker at high levels (Figure 3C, upper panel). On the other hand, HieRFIT classification of these cells demonstrated a clearer

343 separation between these two highly similar subtypes of monocytes while preserving the major

344 monocyte marker expression in all cells even in cells predicted with the label of the parent node,

345 "Monocytes" (Figure 3C, lower panel).

\section{Comparative performance evaluation with intra-dataset tests}

We evaluated the performance of HieRFIT on a large number of different datasets, with varying complexity, technology, and size. These include human and mouse pancreas datasets (Baron et al., 2016, Muraro et al., 2016, Segerstolpe et al., 2016, Xin et al., 2016), human PBMC

350 (Zheng et al., 2017a), human lung cancer cell lines (Tian et al., 2019), mouse cortex and nervous 351 system (Tasic et al., 2018, Zeisel et al., 2018) as well as whole mouse datasets from Tabula Muris

352 consortium (2018) (Supplemental Table 2). We also compared its performance against other cell

353 type classification that use supervised machine learning approaches to create a predictive model

354 based on the training data. The first benchmarking was based on intra-dataset evaluations with 5-

355 fold cross validation. Some of the datasets with multi-level cell type annotations were treated

356 separately as different datasets. We calculated the mean-F1 score of each classification tool as the

357 overall performance averaged across each cell class in the datasets. To obtain a fair benchmarking,

358 we included only leaf node predictions of HieRFIT and excluded the intermediate node

359 classifications in the F1-score computations. 
362 HieRFIT demonstrates better performance than 16 of them (Supplemental Figure 1). LDA,

363 ACTINN, SingleR, SVM, singleCellNet, and SVM with rejection demonstrate comparable performance

364 against HieRFIT (Figure 4A, heatmap). Both SVM and SVM with rejection option perform better

365 HieRFIT on most datasets except two of them. Out of 18 mean-F1 scores of datasets, ACTINN is

366 better on 12 datasets, LDA is 11 , singleCellNet 10, and singleR is better on only 3 datasets compared

367 to HieRFIT. SingleR and singleCellNet fails to complete the tasks on the complex datasets with large

368 number of cell types, such as Zeisel (237) and AMB (92), TM (55), and Zheng datasets. 5 out of these

3696 classification approaches lack an important feature, a rejection option. HieRFIT, along with LDA

370 (with rejection), scClassify, and CHETAH, returned low levels of 'unlabeled' predictions while SVM

371 (with rejection), scmap (both 'cell' or 'cluster' modes), Cell-BLAST, and scID classifications

372 contained high level of 'unlabeled' results (Figure 4A, boxplot). Almost all of the classification tools

373 perform the worst on Zheng PBMC (11 cell types) dataset, likely due to its intrinsic complexity.

We further explored the performance of HieRFIT in depth by comparing it to other two

375 tools, scClassify and CHETAH, with similar hierarchical classification approaches to ours and with

376 the most commonly used software, Seurat. We computed the hierarchical precision, recall, and F-1

377 score, which takes the intermediate cell type predictions into account when computing the

378 performance metric. To be fair to the other tools, we used the same hierarchical tree that HieRFIT

379 used in the computation of the hierarchical metrics for the other tools. We obtained the results for

380 hierarchical precision, recall, and F-1 score measurements from the 18 intra-datasets through 5-

381 fold cross-validations.

HieRFIT and the other three classification tools demonstrate high levels of hierarchical

383 precision in all datasets, >91\%, except 'Zheng' datasets (Figure 4B, upper panel). However,

384 scClassify fails to return the results for AMB (92) and Seurat fails to identify enough significant

385 anchors for "CellBench (CEL-Seq2)" dataset. On the other hand, HieRFIT, returns class predictions 
with consistently high recall rates (> 89\%) for all datasets while scClassify and CHETAH showed significantly lower recalls especially on tasks with complex datasets with large number of cell types

(Figure 4B, middle panel). As the performance metric that takes precision and recall into account,

hierarchical F-1 score clearly demonstrates that HieRFIT performs at consistent levels and comparable to Seurat classifications (Figure 4B, lower panel).

To better evaluate the HieRFIT results in the hierarchical classification context, we categorized the projected cell types based on their positions on the reference tree (Figure 4C).

393 These categories reflect the level of prediction accuracy relative to the hierarchical relationship

394 defined as cell type similarities. These categories are as follows: The projection cell type is

395 categorized as 'Correct node' if it is same as the true cell type (prior), as 'Correct parent' if it is parent of true cell type, as 'Correct ancestral node' if it is on the ancestral path (excluding parent node) of true type, as 'Incorrect sibling' if it is a sibling of true type, and as 'Incorrect clade' is if it is any other node with an unshared parent as true label. Using these schemes, we checked the distributions of categorized HieRFIT projections for each intra-dataset task (Figure 4D). HieRFIT

400 returns a large proportion of correct leaf nodes for the majority of datasets. Even for the complex 401 datasets, such as TM (55), AMB (92), and Zeisel (237), the correct leaf node rates are 95\%, 85\%, 402 and 75\%, respectively, while Zheng PBMC dataset (11 cell type) results in inferior profile due it its 403 complexity with high rates of incorrect sibling and clade assignments. The rates of assignments 404 from other categories are relatively lesser simply due to the intra-dataset tasks using part of the 405 same data to test the performance.

\section{Robustness against various scRNA-seq methods (PBMC bench)}


410 provide more realistic results as they reflect real life usage better. PBMC1 and PBMC2 samples were

411 split into multiple subsets and sequenced with 8 different versions of single-cell RNA-seq methods.

412 In the experiment, we trained the model using data from one method and tested it on datasets

413 generated using other methods (and on the second of the sample pairs in case same methods). As

414 hierarchical precision, recall, and F1-score distributions on all of the combinations, HieRFIT

415 performs consistently well on almost all tasks with average 85\% rates (Figure 5). On the other

416 hand, Seurat and CHETAH, sacrifices extensive precision and recall rates, respectively, on many

417 tests while scClassify performs slightly better than those two. However, HieRFIT outperforms all

418 with the highest hF1-score in average across multiple tasks. Although HieRFIT's worst performance

419 appears to be the model generated with inDrop data when tested on CEL-Seq data as 64\% hF-score,

420 it is still comparably better than its contenders. Overall, these results show that HieRFIT exhibits

421 robustness against various batch effects due to different scRNA-seq methods and performs better

422 than other tools in various aspects. 


\section{Discussion}

425 becoming increasingly difficult as the diversity of single-cell experiments increases. In this study,

426 we attempted to address one of the challenges in the developing field of scRNA-seq with an

427 alternative perspective. Hierarchical classification, as opposed to common flat classifiers, is

428 currently generating more interest in the community because it takes the cell type relationships

429 into account in addition to providing more insight into intermediate cell types (Wu and $\mathrm{Wu}, 2020)$.

430 The hierarchical approach has been used in many other fields including medical sciences

431 (Dimitrovski et al., 2011).

In this work, we hypothesized that hierarchical organization of cell types and class

433 relationships will provide more accurate decisions compared to flat classification approaches. We,

434 then, implemented our approach as a user-friendly R package and evaluated its performance with

435 commonly used public datasets. We demonstrated HieRFIT's better classification of PBMC cell

436 types, even in low abundances, in concordance with their marker gene expression profiles as

437 opposed to manual annotations by widely used software, Seurat. In addition, the performance

438 evaluations against other available single-cell classification tools and machine learning algorithms

439 showed that HieRFIT provided the most reasonably accurate results. HieRFIT's performance stayed

440 stable across various types of datasets produced with different methods while other tools

441 sometimes showed diminished accuracy, in particular, inter-dataset tasks. With its 'divide and

442 conquer' approach, HieRFIT was able handle very complex tasks with a large number of cell types

443 and total cells without any issue.

Furthermore, HieRFIT showed consistently better performance on classification challenges

445 against two other tools, CHETAH and scClassify, which have similar hierarchical classification

446 approaches. Both CHETAH and scClassify learn tree topologies directly from reference data as 
447 CHETAH builds a binary tree of cell types using average linkage distances based on their Spearman

448 correlations while scClassify uses hierarchical ordered partitioning and collapsing hybrid

449 (HOPACH) algorithm for tree construction which allows multi-children nodes. However, both tools

450 define the intermediate cell types with labels that are hard to interpret. HieRFIT on the other hand

451 covers both approaches by providing users an option, in addition to ability to create a de novo tree,

452 to define a tree containing intermediate nodes with meaningful cell labels as opposed to other tools.

We implemented the 'local classifier per parent node' (LCPN) approach in HieRFIT as

454 opposed to the global classifier approach that takes the entire tree topology into a single model.

455 Hierarchical classification implemented in LCPN attitude has been reported to have better accuracy

456 as compared to flat classifiers (Gauch et al., 2009, Jin et al., 2008, Xiao et al., 2007). In addition,

457 using various combinations of different classification algorithms as local classifiers has previously

458 been reported (Secker et al., 2007). One important feature of HieRFIT originates from its 'non-

459 mandatory leaf node prediction' based decision scheme which allows intermediate nodes to be

460 assigned as well. Our decision rule is based on choosing the best scoring node on the tree.

461 Alternative decision approaches have been proposed, e.g. 'sequential boolean decision rule' which

462 chooses child nodes, starting from root, until reaching to a leaf node (Bryant, 1992). However, this

463 approach might be prone to error propagation more than other top-down approaches. The

464 challenge is to properly combine local classifiers so that their unbiased outputs can be used for the

465 decision making process. It is commonly known that machine learning based classifiers are prone

466 to imbalanced class sizes in addition to other intrinsic biases such as batch effect. To account for

467 these, we utilized a certainty function derived from asymmetric entropy which provided precise

468 confidence estimations about class assignment. The path certainty metric accumulates higher

469 scores when correct cell types are picked along the ancestral path while their siblings and out-

470 groups behave as antagonists. Thus, accumulated confidence allows better decision regardless of

471 the complexity of data or tree topology. 
As all other computational approaches, HieRFIT also has several limitations. First of all, it

473 requires a reference data with properly annotated cell types. Although relying on reference data

474 and its cell types can introduce biases due to inconsistencies in annotations, HieRFIT's ensemble

475 based classifiers, random forest, can compensate for subtle fluctuations. Reference based

476 classification approaches usually miss the opportunity to discover novel cell types due to their

477 dependency on prior information. Another limitation of reliance on reference data is that some cell

478 types are represented with low numbers of cells. However, with the fast development of new

479 methods, single-cell based atlas projects provide exponentially increasing datasets. Secondly,

480 HieRFIT relies on a user provided tree, a predefined class hierarchy, and assumes that the tree

481 topology reflects biological cell type relationships with their underlying gene expression profiles in

482 the reference data. To prevent senseless results, users must be cautious about providing a tree

483 topology for classification purposes with HieRFIT. If a user skips to provide a cell type tree, creating

484 a class hierarchy by learning from data (e.g. by hierarchical clustering) can also be limited since

485 similarity driven hierarchy is prone to data specific artifacts and over-fitting.

In this study, we proposed to utilize hierarchical relationships between cell types to better

487 harvest biological information and provide more insight about the cell type identities. HieRFIT

488 provides stable and accurate cell type classification of single-cell RNA-seq data with hierarchical

489 manner. It will contribute to the field not only by providing a new perspective and faster cell type

490 projections from larger atlas projects but also allowing cross comparisons across various datasets

491 effectively. 
bioRxiv preprint doi: https://doi.org/10,1101/2020 09,16,300822: this version posted September 18,2020 The copvriaht holder for this preprint (which was not certified by peer review) is the author/funder, who has granted bioRxiv a license to display the preprint in perpetuity. It is made available under aCC-BY-NC-ND 4.0 International license.

\section{Acknowledgements}

493 Special thanks to Aaron Kitzmiller, Hansaim Lim, and Varenka Rodriguez Diblasi. 


\section{References}

499

500

501

502

503

504

505

506

2018. Single-cell transcriptomics of 20 mouse organs creates a Tabula Muris. Nature, 562, 367-372.

ABDELAAL, T., MICHIELSEN, L., CATS, D., HOOGDUIN, D., MEI, H., REINDERS, M. J. T. \& MAHFOUZ, A. 2019a. A comparison of automatic cell identification methods for single-cell RNA sequencing data. Genome Biology, 20, 194.

ABDELAAL, T., MICHIELSEN, L., CATS, D., HOOGDUIN, D., MEI, H., REINDERS, M. J. T. \& MAHFOUZ, A. 2019b. A comparison of automatic cell identification methods for single-cell RNA sequencing data. Genome Biol., 20, 194.

AEVERMANN, B. D., NOVOTNY, M., BAKKEN, T., MILLER, J. A., DIEHL, A. D., OSUMISUTHERLAND, D., LASKEN, R. S., LEIN, E. S. \& SCHEUERMANN, R. H. 2018. Cell type discovery using single-cell transcriptomics: implications for ontological representation. Human Molecular Genetics, 27, R40-R47.

BARON, M., VERES, A., WOLOCK, SAMUEL L., FAUST, AUBREY L., GAUJOUX, R., VETERE, A., RYU, JENNIFER H., WAGNER, BRIDGET K., SHEN-ORR, SHAI S., KLEIN, ALLON M., MELTON, DOUGLAS A. \& YANAI, I. 2016. A Single-Cell Transcriptomic Map of the Human and Mouse Pancreas Reveals Inter- and Intra-cell Population Structure. Cell Systems, 3, 346-360.e4.

BRYANT, R. E. 1992. Symbolic Boolean manipulation with ordered binary-decision diagrams. ACM Comput. Surv., 24, 293-318.

BUTLER, A., HOFFMAN, P., SMIBERT, P., PAPALEXI, E. \& SATIJA, R. 2018. Integrating singlecell transcriptomic data across different conditions, technologies, and species. Nat. Biotechnol.

CAMPBELL, J. N., MACOSKO, E. Z., FENSELAU, H., PERS, T. H., LYUBETSKAYA, A., TENEN, D., GOLDMAN, M., VERSTEGEN, A. M. J., RESCH, J. M., MCCARROLL, S. A., ROSEN, E. D., LOWELL, B. B. \& TSAI, L. T. 2017. A molecular census of arcuate hypothalamus and median eminence cell types. Nat. Neurosci., 20, 484-496.

CAO, J., PACKER, J. S., RAMANI, V., CUSANOVICH, D. A., HUYNH, C., DAZA, R., QIU, X., LEE, C., FURLAN, S. N., STEEMERS, F. J., ADEY, A., WATERSTON, R. H., TRAPNELL, C. \& SHENDURE, J. 2017. Comprehensive single-cell transcriptional profiling of a multicellular organism. Science, 357, 661-667.

CAO, J., SPIELMANN, M., QIU, X., HUANG, X., IBRAHIM, D. M., HILL, A. J., ZHANG, F., MUNDLOS, S., CHRISTIANSEN, L., STEEMERS, F. J., TRAPNELL, C. \& SHENDURE, J. 2019. The single-cell transcriptional landscape of mammalian organogenesis. Nature, 1.

DE KANTER, J. K., LIJNZAAD, P., CANDELLI, T., MARGARITIS, T. \& HOLSTEGE, F. C. P. 2019. CHETAH: a selective, hierarchical cell type identification method for single-cell RNA sequencing. Nucleic Acids Research, 47, e95-e95.

DIMITROVSKI, I., KOCEV, D., LOSKOVSKA, S. \& DŽEROSKI, S. 2011. Hierarchical annotation of medical images. Pattern Recognit., 44, 2436-2449.

DING, J., ADICONIS, X., SIMMONS, S. K., KOWALCZYK, M. S., HESSION, C. C., MARJANOVIC, N. D., HUGHES, T. K., WADSWORTH, M. H., BURKS, T., NGUYEN, L. T., KWON, J. Y. H., BARAK, B., GE, W., KEDAIGLE, A. J., CARROLL, S., LI, S., HACOHEN, N., ROZENBLATT- 
ROSEN, O., SHALEK, A. K., VILLANI, A.-C., REGEV, A. \& LEVIN, J. Z. 2019. Systematic comparative analysis of single cell RNA-sequencing methods. bioRxiv, 632216.

GAUCH, S., CHANDRAMOULI, A. \& RANGANATHAN, S. 2009. Training a hierarchical classifier using inter document relationships. J. Am. Soc. Inf. Sci., 60, 47-58.

JIN, B., MULLER, B., ZHAI, C. \& LU, X. 2008. Multi-label literature classification based on the Gene Ontology graph. BMC Bioinformatics, 9, 525.

KIRITCHENKO, S., MATWIN, S. \& FAZEL FAMILI, A. 2005. Functional annotation of genes using hierarchical text categorization. in Proc. of the BioLINK SIG: Linking Literature, Information and Knowledge for Biology (held at ISMB-05.

KISELEV, V. Y., ANDREWS, T. S. \& HEMBERG, M. 2019. Challenges in unsupervised clustering of single-cell RNA-seq data. Nature Reviews Genetics, 20, 273-282.

KISELEV, V. Y., KIRSCHNER, K., SCHAUB, M. T., ANDREWS, T., YIU, A., CHANDRA, T., NATARAJAN, K. N., REIK, W., BARAHONA, M., GREEN, A. R. \& HEMBERG, M. 2017. SC3: consensus clustering of single-cell RNA-seq data. Nature Methods, 14, 483-486.

LÄHNEMANN, D., KÖSTER, J., SZCZUREK, E., MCCARTHY, D. J., HICKS, S. C., ROBINSON, M. D., VALLEJOS, C. A., CAMPBELL, K. R., BEERENWINKEL, N., MAHFOUZ, A., PINELLO, L., SKUMS, P., STAMATAKIS, A., ATTOLINI, C. S.-O., APARICIO, S., BAAIJENS, J., BALVERT, M., BARBANSON, B. D., CAPPUCCIO, A., CORLEONE, G., DUTILH, B. E., FLORESCU, M., GURYEV, V., HOLMER, R., JAHN, K., LOBO, T. J., KEIZER, E. M., KHATRI, I., KIELBASA, S. M., KORBEL, J. O., KOZLOV, A. M., KUO, T.-H., LELIEVELDT, B. P. F., MANDOIU, I. I., MARIONI, J. C., MARSCHALL, T., MÖLDER, F., NIKNEJAD, A., RACZKOWSKI, L., REINDERS, M., RIDDER, J. D., SALIBA, A.-E., SOMARAKIS, A., STEGLE, O., THEIS, F. J., YANG, H., ZELIKOVSKY, A., MCHARDY, A. C., RAPHAEL, B. J., SHAH, S. P. \& SCHÖNHUTH, A. 2020. Eleven grand challenges in single-cell data science. Genome Biol., 21, 31.

LIN, Y., CAO, Y., KIM, H. J., SALIM, A., SPEED, T. P., LIN, D., YANG, P. \& YANG, J. Y. H. 2019. scClassify: hierarchical classification of cells. bioRxiv, 776948.

MA, F. \& PELLEGRINI, M. 2019. ACTINN: automated identification of cell types in single cell RNA sequencing. Bioinformatics, 36, 533-538.

MACAULAY, I. C., SVENSSON, V., LABALETTE, C., FERREIRA, L., HAMEY, F., VOET, T., TEICHMANN, S. A. \& CVEJIC, A. 2016. Single-Cell RNA-Sequencing Reveals a Continuous Spectrum of Differentiation in Hematopoietic Cells. Cell Rep., 14, 966977.

MARCELLIN, S., ZIGHED, D. A. \& RITSCHARD, G. 2006. An asymmetric entropy measure for decision trees.

MURARO, M. J., DHARMADHIKARI, G., GRÜN, D., GROEN, N., DIELEN, T., JANSEN, E., VAN GURP, L., ENGELSE, M. A., CARLOTTI, F., DE KONING, E. J. \& VAN OUDENAARDEN, A. 2016. A Single-Cell Transcriptome Atlas of the Human Pancreas. Cell Syst, 3, 385394.e3.

PETEGROSSO, R., LI, Z. \& KUANG, R. 2020. Machine learning and statistical methods for clustering single-cell RNA-sequencing data. Brief Bioinform, 21, 1209-1223.

PLASSCHAERT, L. W., ŽILIONIS, R., CHOO-WING, R., SAVOVA, V., KNEHR, J., ROMA, G., KLEIN, A. M. \& JAFFE, A. B. 2018. A single-cell atlas of the airway epithelium reveals the CFTR-rich pulmonary ionocyte. Nature, 560, 377-381.

PLATT, J. C. 1999. Probabilistic Outputs for Support Vector Machines and Comparisons to Regularized Likelihood Methods. ADVANCES IN LARGE MARGIN CLASSIFIERS. 
PLINER, H. A., SHENDURE, J. \& TRAPNELL, C. 2019. Supervised classification enables rapid annotation of cell atlases. Nature Methods, 16, 983-986.

ROSENBERG, A. B., ROCO, C. M., MUSCAT, R. A., KUCHINA, A., SAMPLE, P., YAO, Z., GRAYBUCK, L. T., PEELER, D. J., MUKHERJEE, S., CHEN, W., PUN, S. H., SELLERS, D. L., TASIC, B. \& SEELIG, G. 2018. Single-cell profiling of the developing mouse brain and spinal cord with split-pool barcoding. Science, 360, 176-182.

SECKER, A. D., DAVIES, M. N., FREITAS, A. A., TIMMIS, J., MENDAO, M. \& FLOWER, D. R. 2007. An experimental comparison of classification algorithms for hierarchical prediction of protein function. Expert Update (Magazine of the British Computer Society's Specialist Group on AI), 9, 17-22.

SEGERSTOLPE, Å., PALASANTZA, A., ELIASSON, P., ANDERSSON, E. M., ANDRÉASSON, A. C., SUN, X., PICELLI, S., SABIRSH, A., CLAUSEN, M., BJURSELL, M. K., SMITH, D. M., KASPER, M., ÄMMÄLÄ, C. \& SANDBERG, R. 2016. Single-Cell Transcriptome Profiling of Human Pancreatic Islets in Health and Type 2 Diabetes. Cell Metab, 24, 593-607.

SUO, S., ZHU, Q., SAADATPOUR, A., FEI, L., GUO, G. \& YUAN, G.-C. 2018. Revealing the Critical Regulators of Cell Identity in the Mouse Cell Atlas. Cell Reports, 25, 1436-1445.e3.

TABULA MURIS, C., OVERALL, C., LOGISTICAL, C., ORGAN, C., PROCESSING, LIBRARY, P., SEQUENCING, COMPUTATIONAL DATA, A., CELL TYPE, A., WRITING, G., SUPPLEMENTAL TEXT WRITING, G. \& PRINCIPAL, I. 2018. Single-cell transcriptomics of 20 mouse organs creates a Tabula Muris. Nature, 562, 367-372.

TAN, Y. \& CAHAN, P. 2019. SingleCellNet: A Computational Tool to Classify Single Cell RNASeq Data Across Platforms and Across Species. Cell Systems, 9, 207-213.e2.

TANG, M., KAYMAZ, Y., LOGEMAN, B., EICHHORN, S., LIANG, Z. S., DULAC, C. \& SACKTON, T. B. 2020. Evaluating single-cell cluster stability using the Jaccard similarity index. bioRxiv.

TASIC, B., YAO, Z., GRAYBUCK, L. T., SMITH, K. A., NGUYEN, T. N., BERTAGNOLLI, D., GOLDY, J., GARREN, E., ECONOMO, M. N., VISWANATHAN, S., PENN, O., BAKKEN, T., MENON, V., MILLER, J., FONG, O., HIROKAWA, K. E., LATHIA, K., RIMORIN, C., TIEU, M., LARSEN, R., CASPER, T., BARKAN, E., KROLL, M., PARRY, S., SHAPOVALOVA, N. V., HIRSCHSTEIN, D., PENDERGRAFT, J., SULLIVAN, H. A., KIM, T. K., SZAFER, A., DEE, N., GROBLEWSKI, P., WICKERSHAM, I., CETIN, A., HARRIS, J. A., LEVI, B. P., SUNKIN, S. M., MADISEN, L., DAIGLE, T. L., LOOGER, L., BERNARD, A., PHILLIPS, J., LEIN, E., HAWRYLYCZ, M., SVOBODA, K., JONES, A. R., KOCH, C. \& ZENG, H. 2018. Shared and distinct transcriptomic cell types across neocortical areas. Nature, 563, 72-78.

TIAN, L., DONG, X., FREYTAG, S., KA, L. C., SU, S., JALALABADI, A., AMANN-ZALCENSTEIN, D., WEBER, T. S., SEIDI, A., JABBARI, J. S., NAIK, S. H. \& RITCHIE, M. E. 2019.

Benchmarking single cell RNA-sequencing analysis pipelines using mixture control experiments. Nat Methods, 16, 479-487.

WILBREY-CLARK, A., ROBERTS, K. \& TEICHMANN, S. A. 2020. Cell Atlas technologies and insights into tissue architecture. Biochemical Journal, 477, 1427-1442.

WU, Z. \& WU, H. 2020. Accounting for cell type hierarchy in evaluating single cell RNA-seq clustering. Genome Biol, 21, 123.

XIAO, Z., DELLANDREA, E., DOU, W. \& CHEN, L. 2007. Hierarchical classification of emotional speech. IEEE Trans. Multimedia. 
XIN, Y., KIM, J., OKAMOTO, H., NI, M., WEI, Y., ADLER, C., MURPHY, A. J., YANCOPOULOS, G. D., LIN, C. \& GROMADA, J. 2016. RNA Sequencing of Single Human Islet Cells Reveals Type 2 Diabetes Genes. Cell Metab, 24, 608-615.

ZEISEL, A., HOCHGERNER, H., LÖNNERBERG, P., JOHNSSON, A., MEMIC, F., VAN DER ZWAN, J., HÄRING, M., BRAUN, E., BORM, L. E., LA MANNO, G., CODELUPPI, S., FURLAN, A., LEE, K., SKENE, N., HARRIS, K. D., HJERLING-LEFFLER, J., ARENAS, E., ERNFORS, P., MARKLUND, U. \& LINNARSSON, S. 2018. Molecular Architecture of the Mouse Nervous System. Cell, 174, 999-1014.e22.

ZHENG, G. X., TERRY, J. M., BELGRADER, P., RYVKIN, P., BENT, Z. W., WILSON, R., ZIRALDO, S. B., WHEELER, T. D., MCDERMOTT, G. P., ZHU, J., GREGORY, M. T., SHUGA, J., MONTESCLAROS, L., UNDERWOOD, J. G., MASQUELIER, D. A., NISHIMURA, S. Y., SCHNALL-LEVIN, M., WYATT, P. W., HINDSON, C. M., BHARADWAJ, R., WONG, A., NESS, K. D., BEPPU, L. W., DEEG, H. J., MCFARLAND, C., LOEB, K. R., VALENTE, W. J., ERICSON, N. G., STEVENS, E. A., RADICH, J. P., MIKKELSEN, T. S., HINDSON, B. J. \& BIELAS, J. H. 2017a. Massively parallel digital transcriptional profiling of single cells. Nat Commun, 8, 14049.

ZHENG, G. X. Y., TERRY, J. M., BELGRADER, P., RYVKIN, P., BENT, Z. W., WILSON, R., ZIRALDO, S. B., WHEELER, T. D., MCDERMOTT, G. P., ZHU, J., GREGORY, M. T., SHUGA, J., MONTESCLAROS, L., UNDERWOOD, J. G., MASQUELIER, D. A., NISHIMURA, S. Y., SCHNALL-LEVIN, M., WYATT, P. W., HINDSON, C. M., BHARADWAJ, R., WONG, A., NESS, K. D., BEPPU, L. W., DEEG, H. J., MCFARLAND, C., LOEB, K. R., VALENTE, W. J., ERICSON, N. G., STEVENS, E. A., RADICH, J. P., MIKKELSEN, T. S., HINDSON, B. J. \& BIELAS, J. H. 2017b. Massively parallel digital transcriptional profiling of single cells. Nat. Commun., 8, 14049.

ZIGHED, D. A., RITSCHARD, G. \& MARCELLIN, S. 2010. Asymmetric and Sample Size Sensitive Entropy Measures for Supervised Learning. In: RAS, Z. W. \& TSAY, L.-S. (eds.) Advances in Intelligent Information Systems. Berlin, Heidelberg: Springer Berlin Heidelberg.

ZUR, R. M., JIANG, Y., PESCE, L. L. \& DRUKKER, K. 2009. Noise injection for training artificial neural networks: a comparison with weight decay and early stopping. Medical physics, 36, 4810-4818. 


\section{Figure Legends:}

665

666

667

668

669

670

671

672

673

674

675

676

677

678

679

680

681

682

683

684

685

686

687

Figure 1. HieRFIT workflow overview. Reference model generation and query

\section{projections.}

Overview of HieRFIT reference model generation and prediction of a query cell class. A) Main process starts with obtaining a tree as a user input or creating from the data. The steps for generating the reference model with a hierarchical tree: 1. Pick an internal node $i$ (i.e. node "B") on the tree, 2. Re-group its children nodes and create an outgroup node for it, 3 . Extract the input expression data based on new group labels for the node, 4. Perform Principal Component Analysis and pick the components that separate the class labels for variable feature selection, $\mathbf{5}$. perform Wilcoxon Rank sum test to determine differentially expressed features, $\mathbf{6}$. Train a local classifier (Random forest) with the group labels and the expression matrix with selected features. Repeat the process until all node classifiers are constructed. B) Query of a test cell and certainty calculations. Given an array of feature expressions of the query cell, the first step is to compute the certainty array $(U)$ for the candidate classes. Votes are collected from each node $i$ (i.e. node "B") for both observed query data and its shuffled data separately. Votes are converted to probabilities using sigmoid calibration with multinomial logistic regression. Using the probability centroids $\left(w_{i}\right)$ as the outcome of the randomized array and the observed probabilities $\left(p_{i}\right)$, compute the certainty value of each class of the node (i.e the certainty of class "E" is 0.24 ). Repeat the process for every class of all internal nodes. C) Determining the cell type/class of a query cell. Step 1: Path certainty scores of each candidate class are computed using the certainty values of nodes for the given query by traversing the tree. The sum of certainty values of outgroup and sibling nodes along the path (nodes in gray) are summed and subtracted from the sum of Certainty values of nodes on the path (nodes in green). Step 2: As the final step, scores are evaluated and the maximum scoring class is returned as the outcome. If none of the classes passes the threshold, $\alpha$, "Undetermined" is returned. 


\section{Figure 2. Demonstration of HieRFIT usage on a PBMC dataset.}

A) The cell type tree used in HieRFIT reference model with 68K-PBMC data. B) The UMAP representation of 3K-PBMC data from 10X Genomics. Cells are colored with cell types which were

691 identified through Seurat clustering and marker expressions (left), cells are colored with HieRFIT

692 reference cell types along with intermediate types specified in the tree file (right). C) Alluvial

693 diagram demonstrating the cross comparisons of HieRFIT projections with the Seurat cell type

694 labels. Each line connecting the two vertical black columns (left bar: prior labels, right bar:

695 projections) represent a cell and are colored based on its HieRFIT projection type. Annotations with

696 less than 1\%, 'HSC' and 'Monocyte progenitor' were not shown.

Figure 3. Concordance analysis of Seurat and HieRFIT classifications with gene expression of cells

A) The heatmap representation of the confusion matrix that summarized the projection results of the 3K-PBMC query data with percent distribution among the tree node labels. B) Violin plots of CD8A and CD8B genes and their co-expression values projected on UMAP representation of cells classified as "Naïve CD4 T cells" by Seurat while HieRFIT predicts them as CD8 cells or its subtypes

703 (upper panel). Similarly, violin plots and co-expression values of IL7R and CCR7 genes projected on 704 UMAP representation of cells predicted as CD4 T cells or its subtypes by HieRFIT in concordance 705 with Seurat (lower panel). C) Normalized expression distribution of three marker genes, "LYZ" and 706 "FCGR3A (CD16)", markers of "monocytes" and subset "CD16 monocytes", respectively, among the 707 cells classified as “CD14+ Monocytes” by Seurat (upper panel). Similar violin plots for expression 708 distribution of the same set of cells grouped based on HieRFIT projections (lower panel). 
Figure 4. Performance on various types of datasets. Robustness against batch biases.

711 Performance results on various types of datasets and comparative benchmarking against other cell

712 type classification tools. A) A heatmap representing the mean F1 scores of each test dataset for the

713 classification tools. The number of cell types of each dataset is shown below the columns. Failed

714 tests without a score are grayed-out. Percent unlabeled data distribution from each test data is

715 shown with an adjacent box plot for each tool. Asterisk (*): Classification tools with rejection

716 option. B) Hierarchical precision (red), recall (cyan), and F-score (green) metrics for the tools

717 HieRFIT, scClassify, CHETAH, and Seurat. C) Various categories of projected cell types by HieRFIT

718 based on their position on tree relative to the prior label. In addition to categories in the table,

719 "Correct children" or "Correct grandchildren" categories are also possible in case of a correct sub-

720 level type assignment. Bar plot summarizes the distribution of these categories for HieRFIT outputs

721 among all test datasets above. D) Stacked-bar plot summarizes the distribution of projection

722 categories for HieRFIT outputs among all test datasets above.

\section{Figure 5. Robustness against batch biases.}

724 Hierarchical precision, recall, and F1-score values of HieRFIT and 3 tools for comparing the

725 performances in various batches with inter-dataset tests. At each iteration, a dataset from paired

726 PBMCs produced with a scRNA method was used to generate the reference model and tested on the

727 second pair of the PBMCs. 


\section{Supplemental Tables}

733 Supplemental Table 1: An example tab-separated cell type table to be used as an input for tree

734 construction and creating a reference model (referencing 68K PBMC dataset). Each row specifies all

735 ancestral/intermediate cell types of each reference cell type (leaves at the end of rows).

736 Supplemental Table 2: Datasets used in the benchmarking analysis.

\section{Supplemental Figures}

739 Supplemental Figure 1. Boxplot for mean F1 scores distribution of each classification tool as an

740 outcome of 18 datasets with 5-fold cross-validation tests (upper plot). Percent unlabeled data

741 distribution from each test data (middle plot). A heatmap showing the Mean F1 scores of each test

742 dataset for the classification tools (lower panel). Failed tests without a score are grayed-out. 


\section{Figure 1}

A
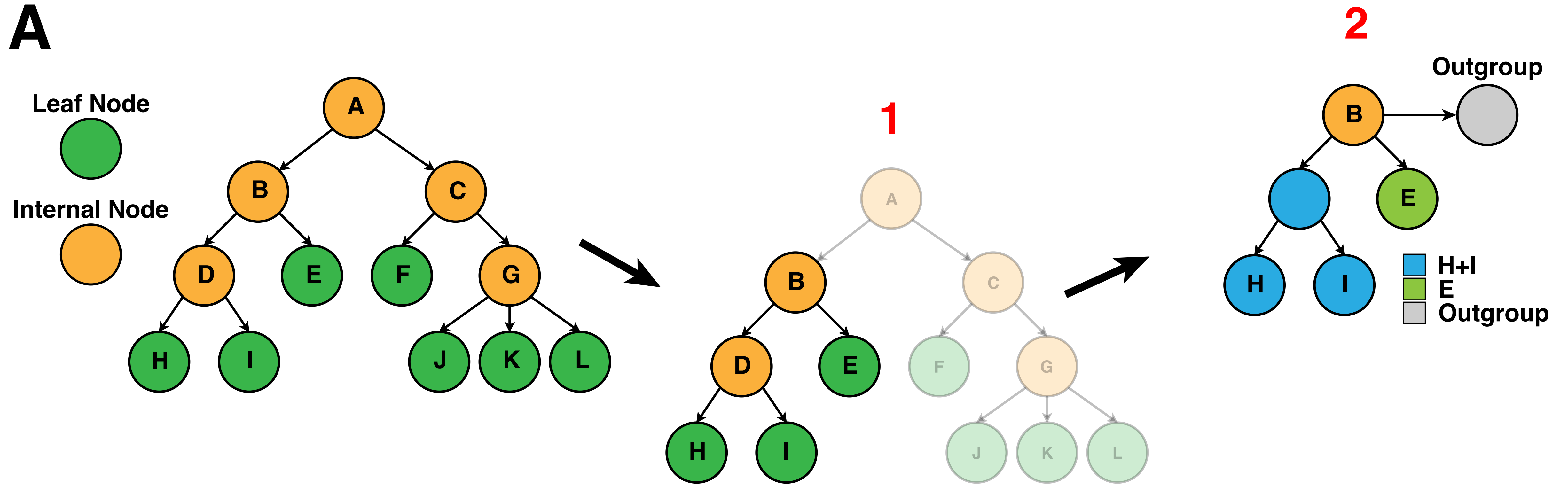

Start with a tree:

1. Pick an internal node,

2. Create an outgroup,

3. Isolate \& regroup data

4. Do PCA \& select PCs,

5. Select features w/ wilcox,

6. Generate a local classifier,

Repeat from 1 until tree completes.
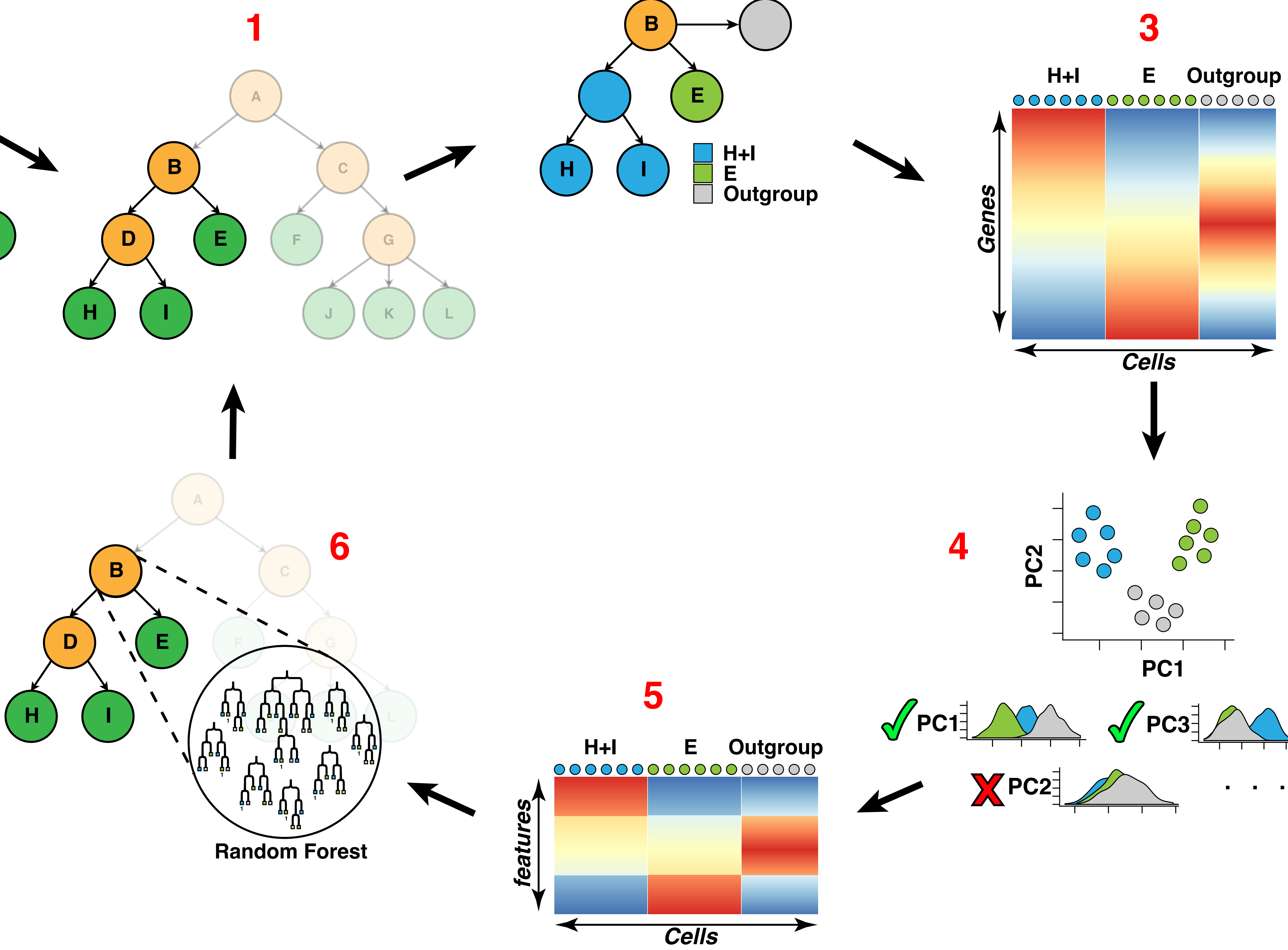

B

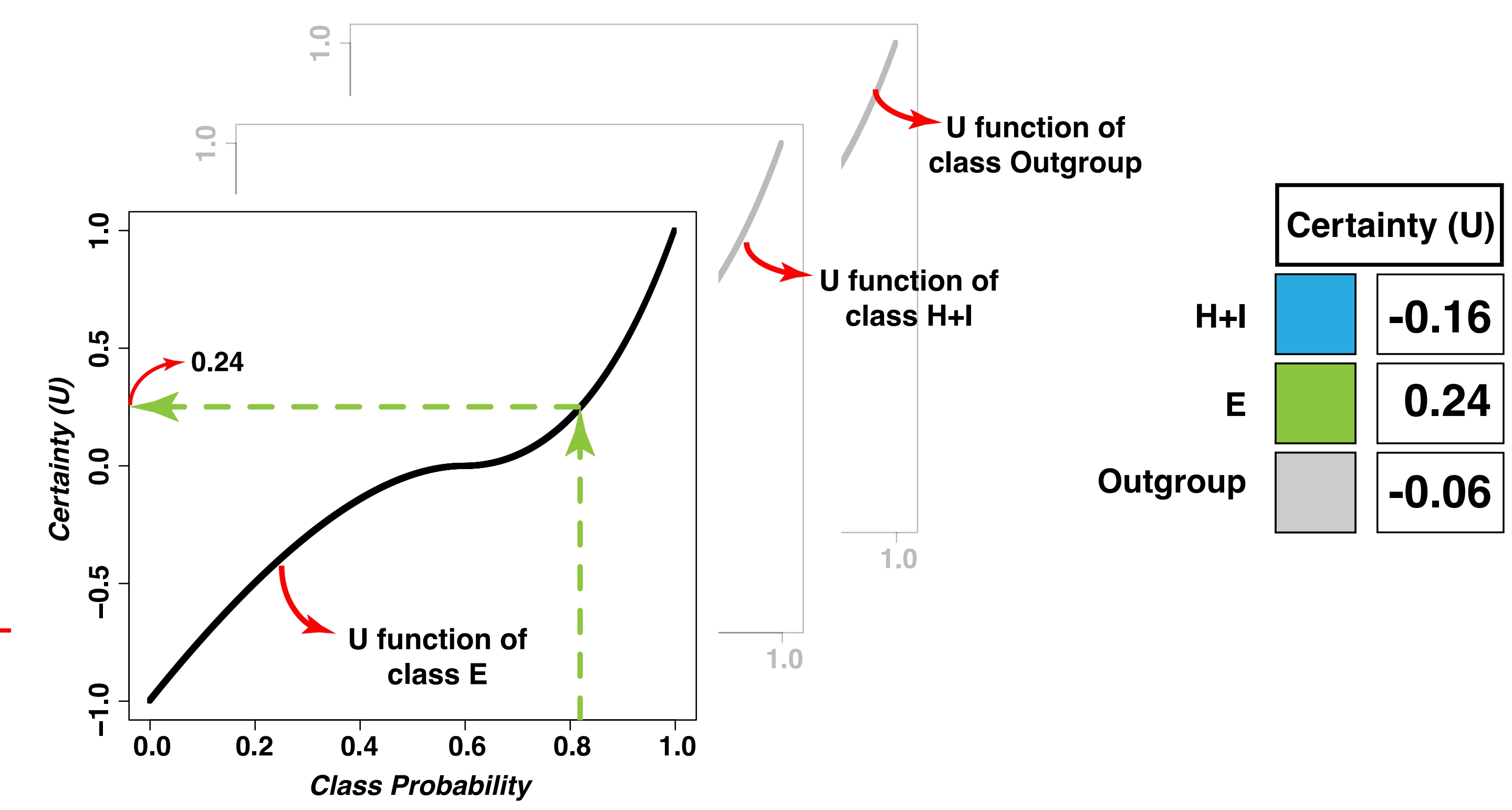

C

1. Calculate Path Certainty Scores of each node

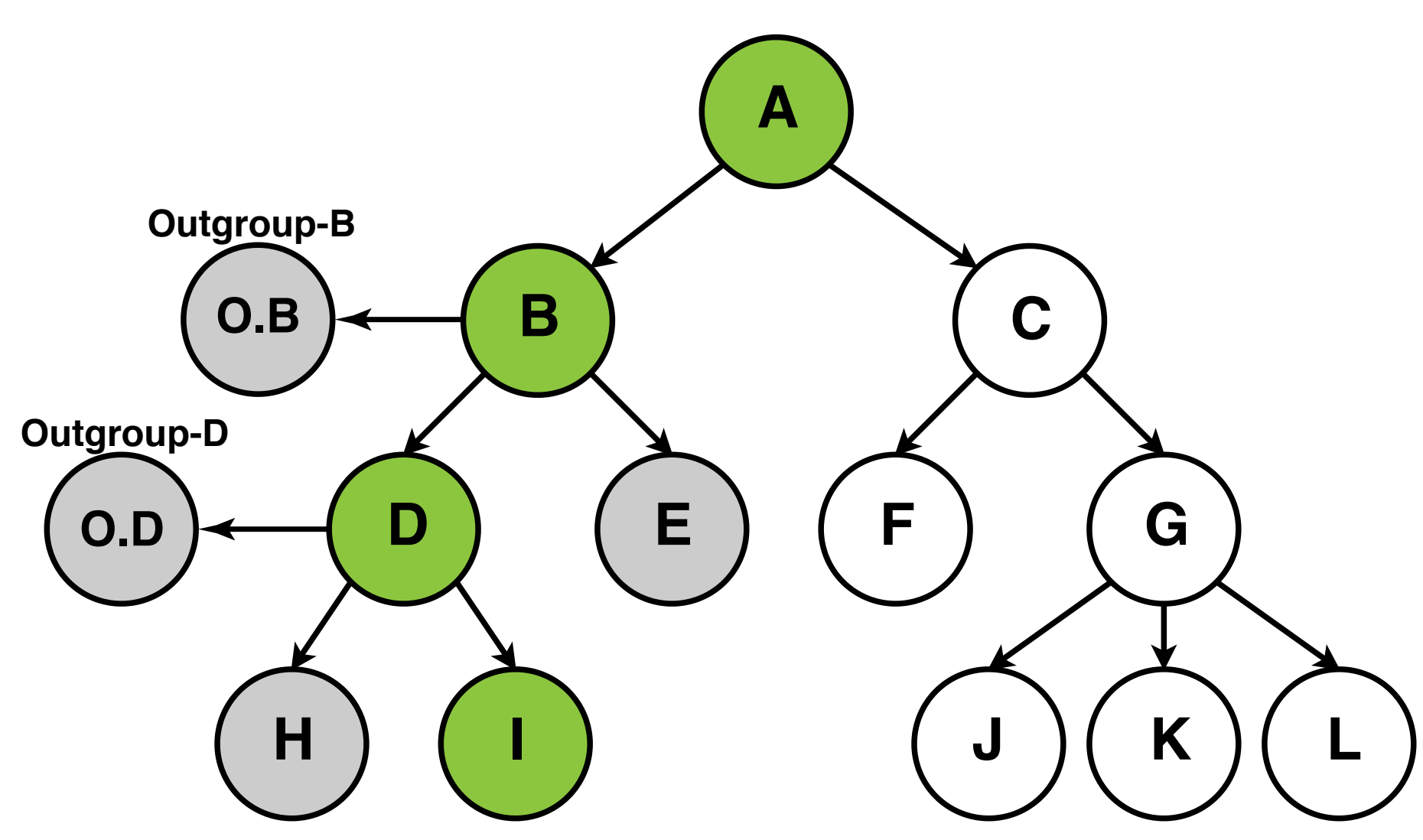

Path Certainty

Score $=A+B+D+I-O . B+O . D+H+E$

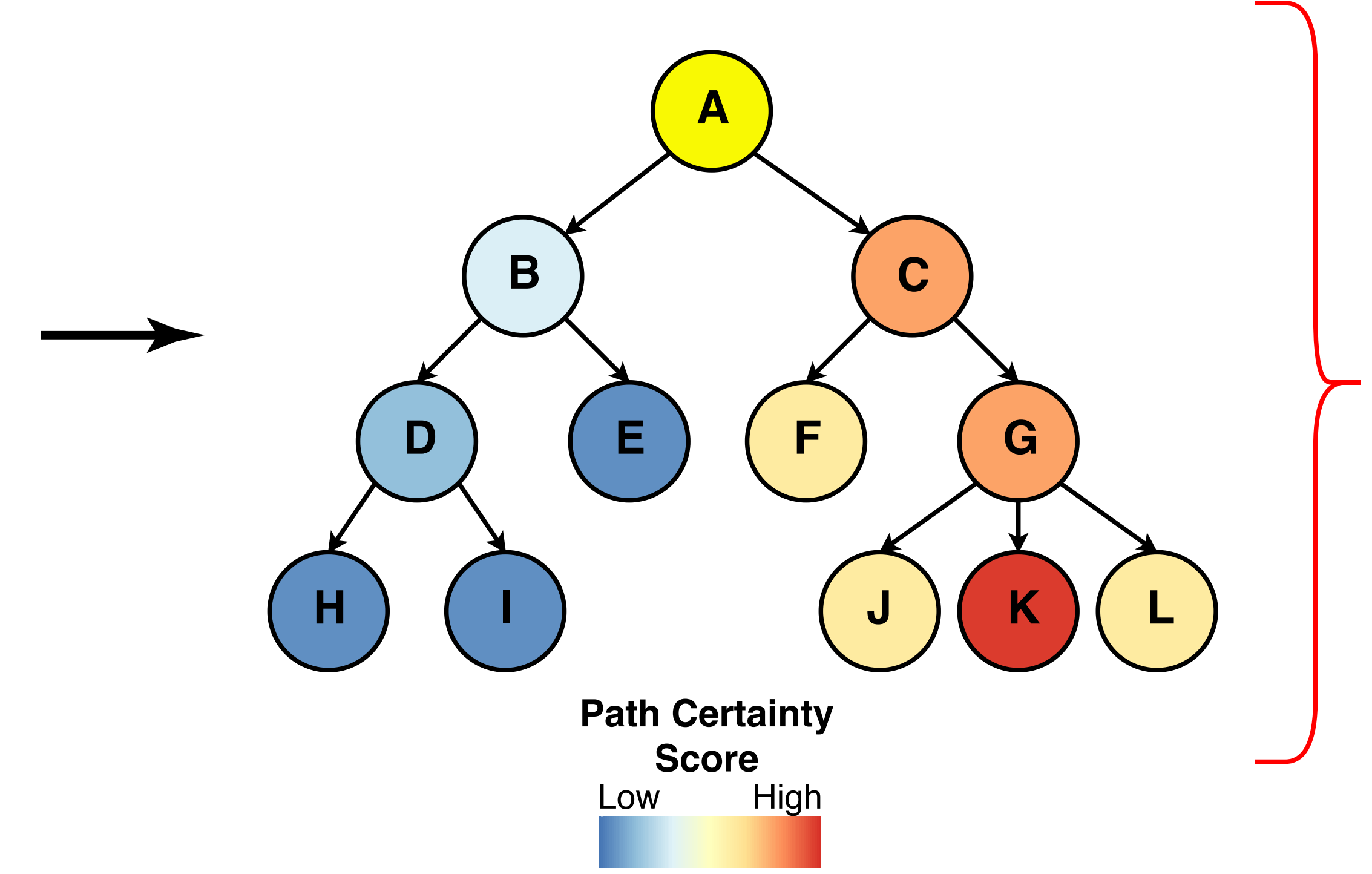

2. Choose the class with highest Path certainty score

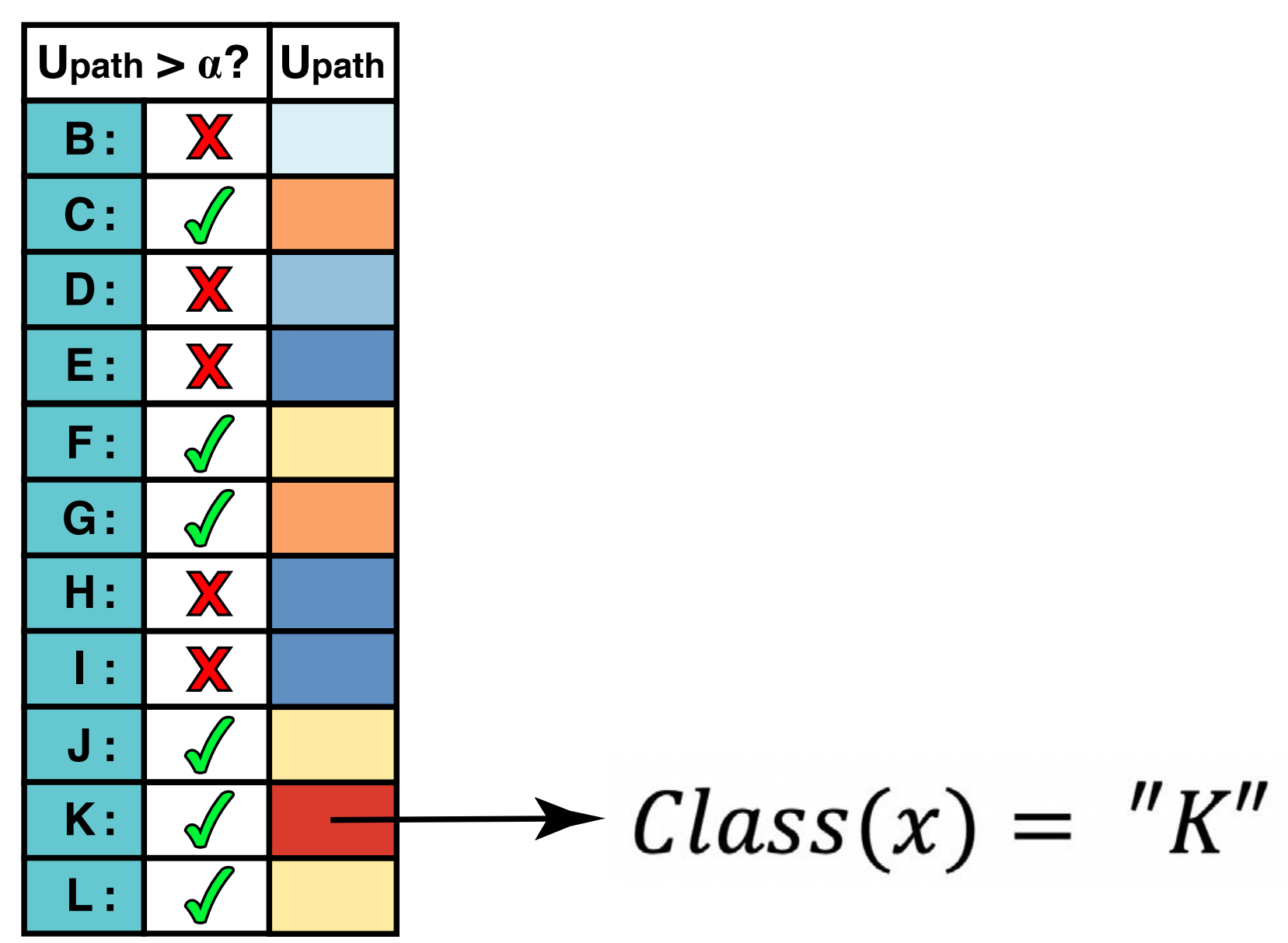




\section{Figure 2}

\section{A}

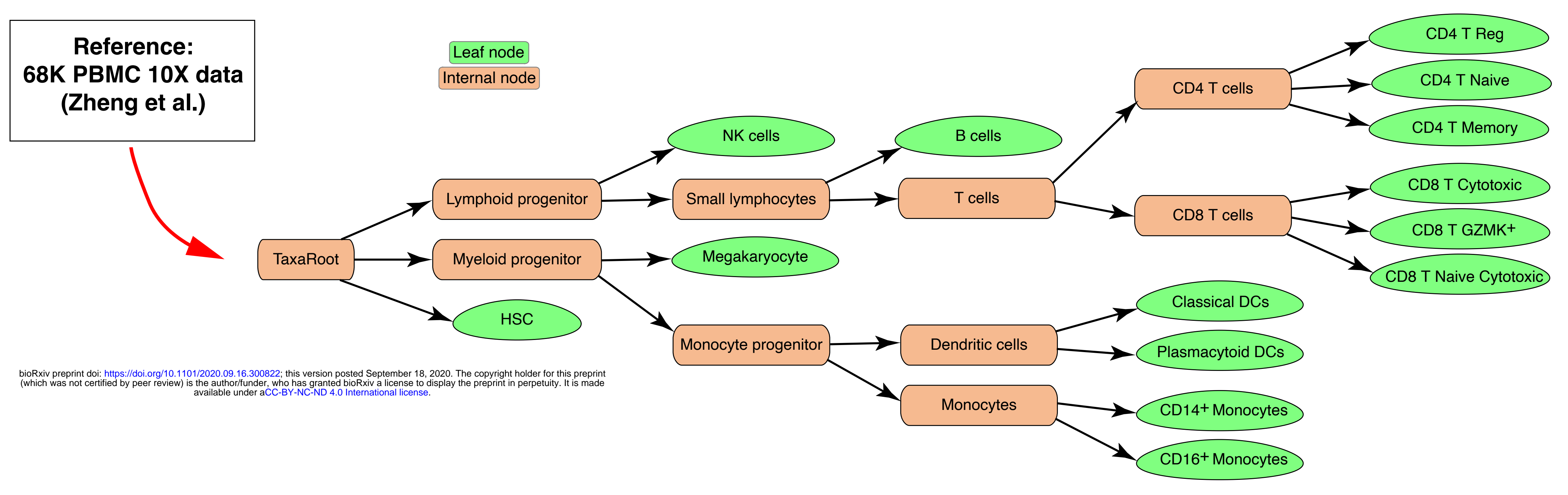

B

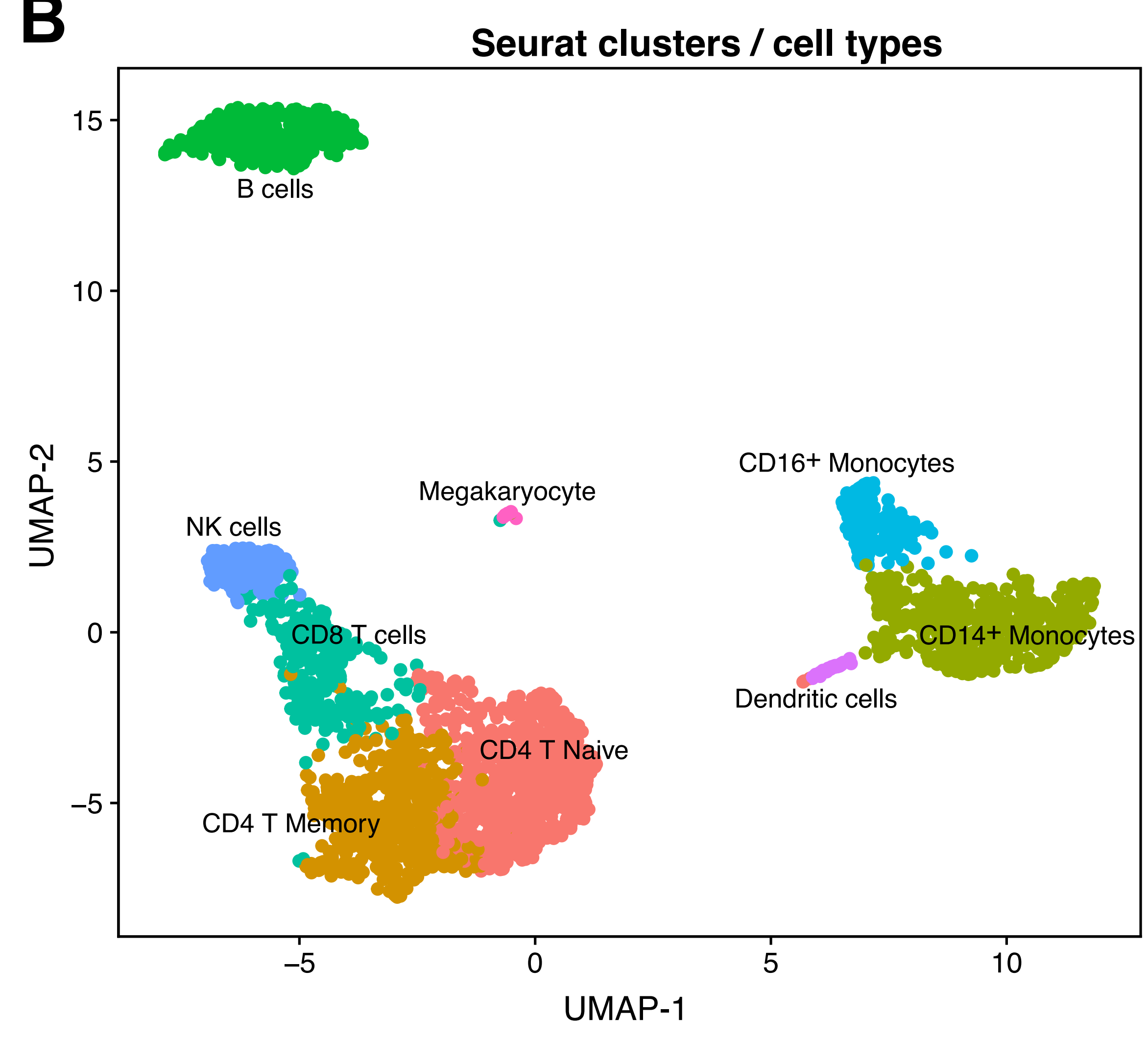

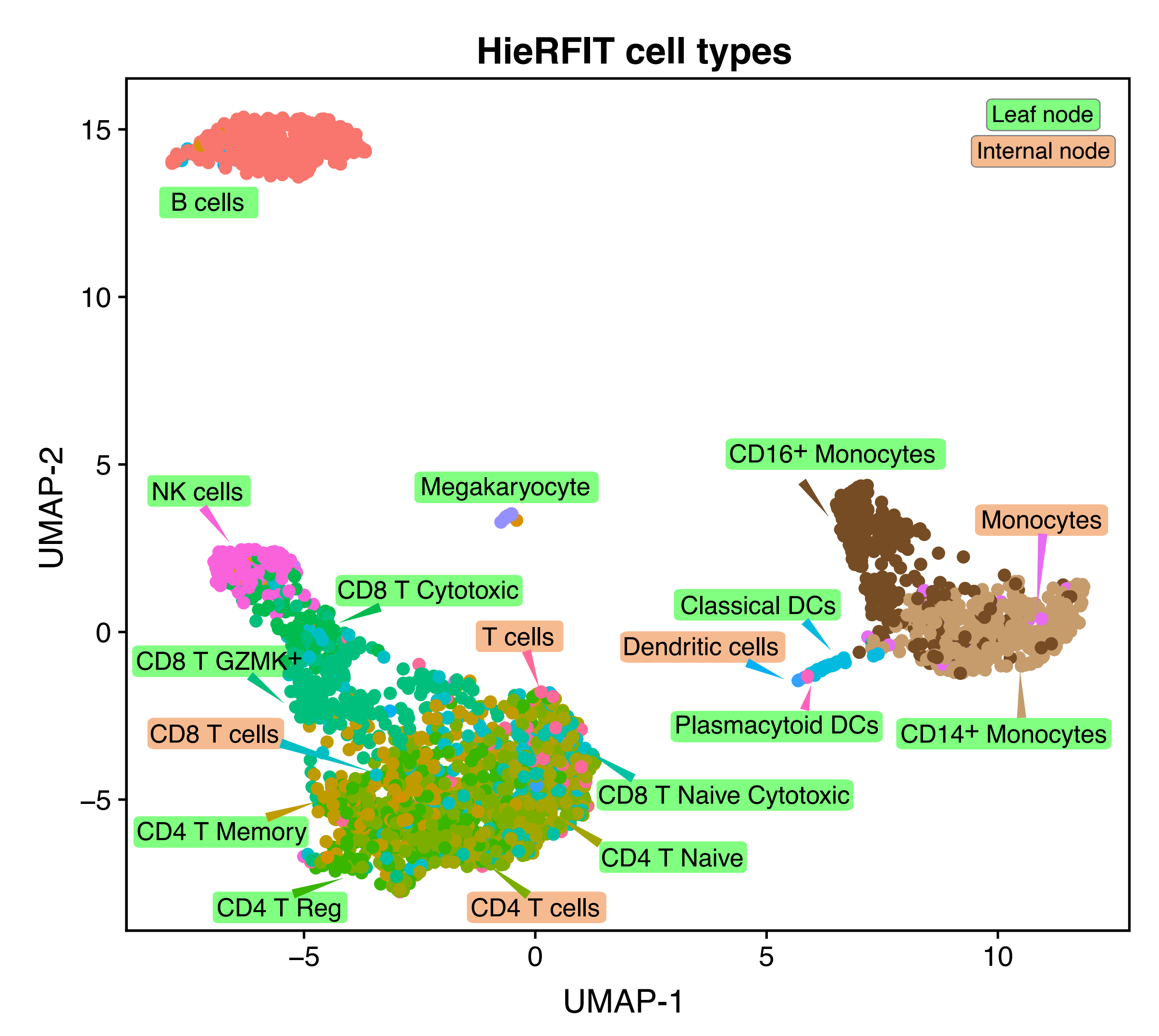

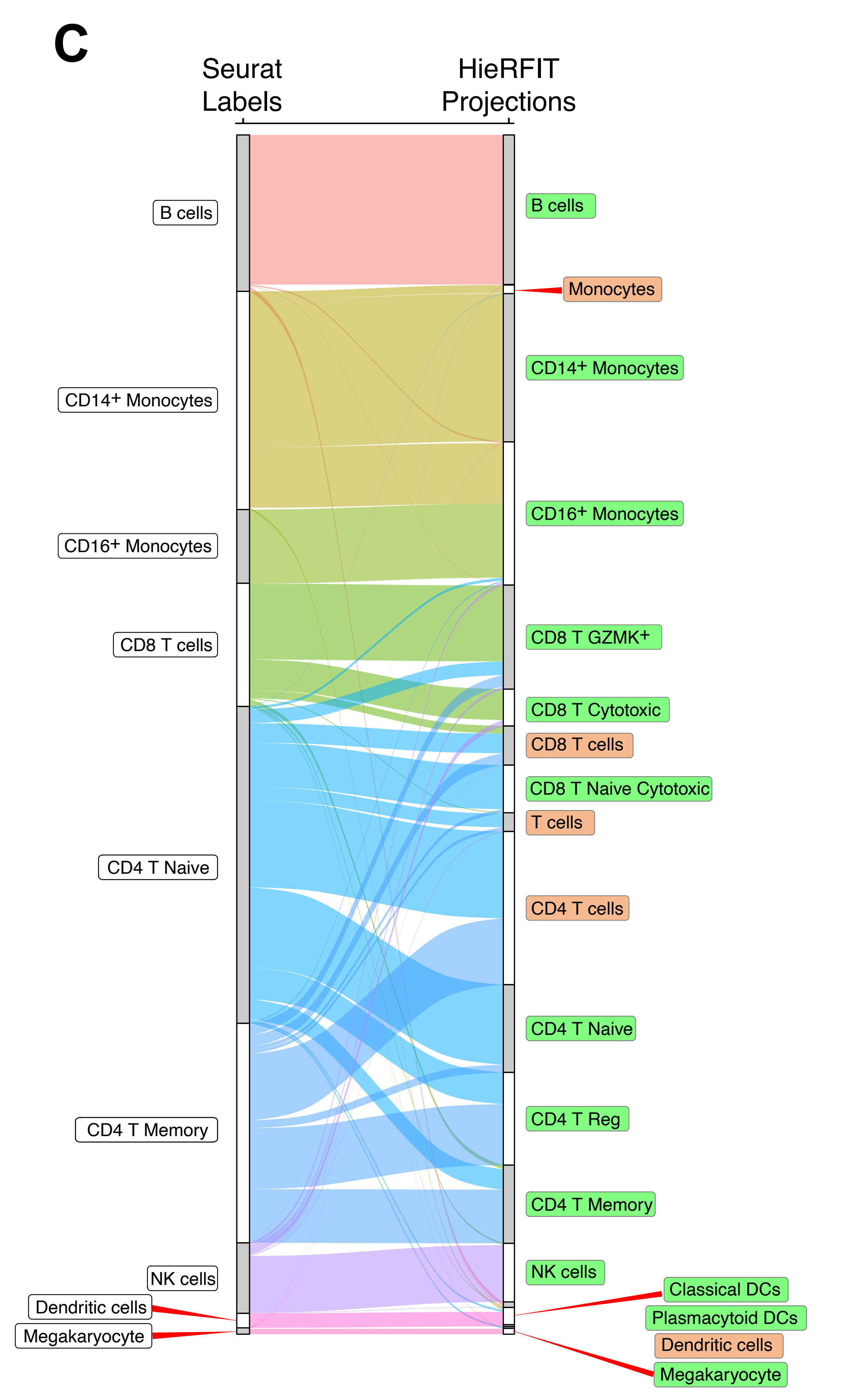




\section{Figure 3}

A

A

\section{B}

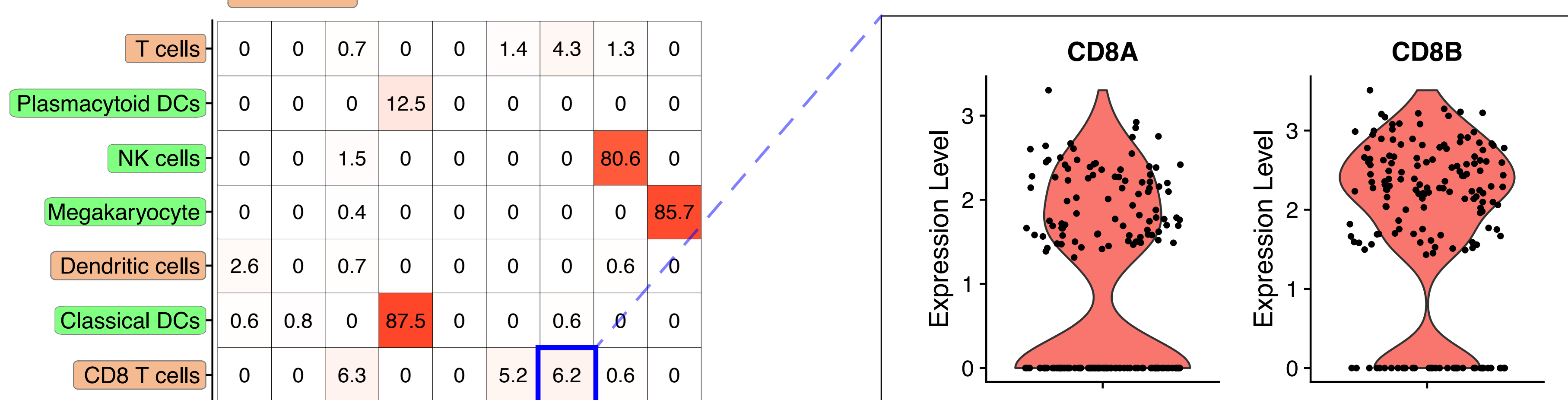

Seurat: CD4 T Naive

HieRFIT: CD8 T cells, CD8 T Naive Cytotoxic, CD8 T GZMK+

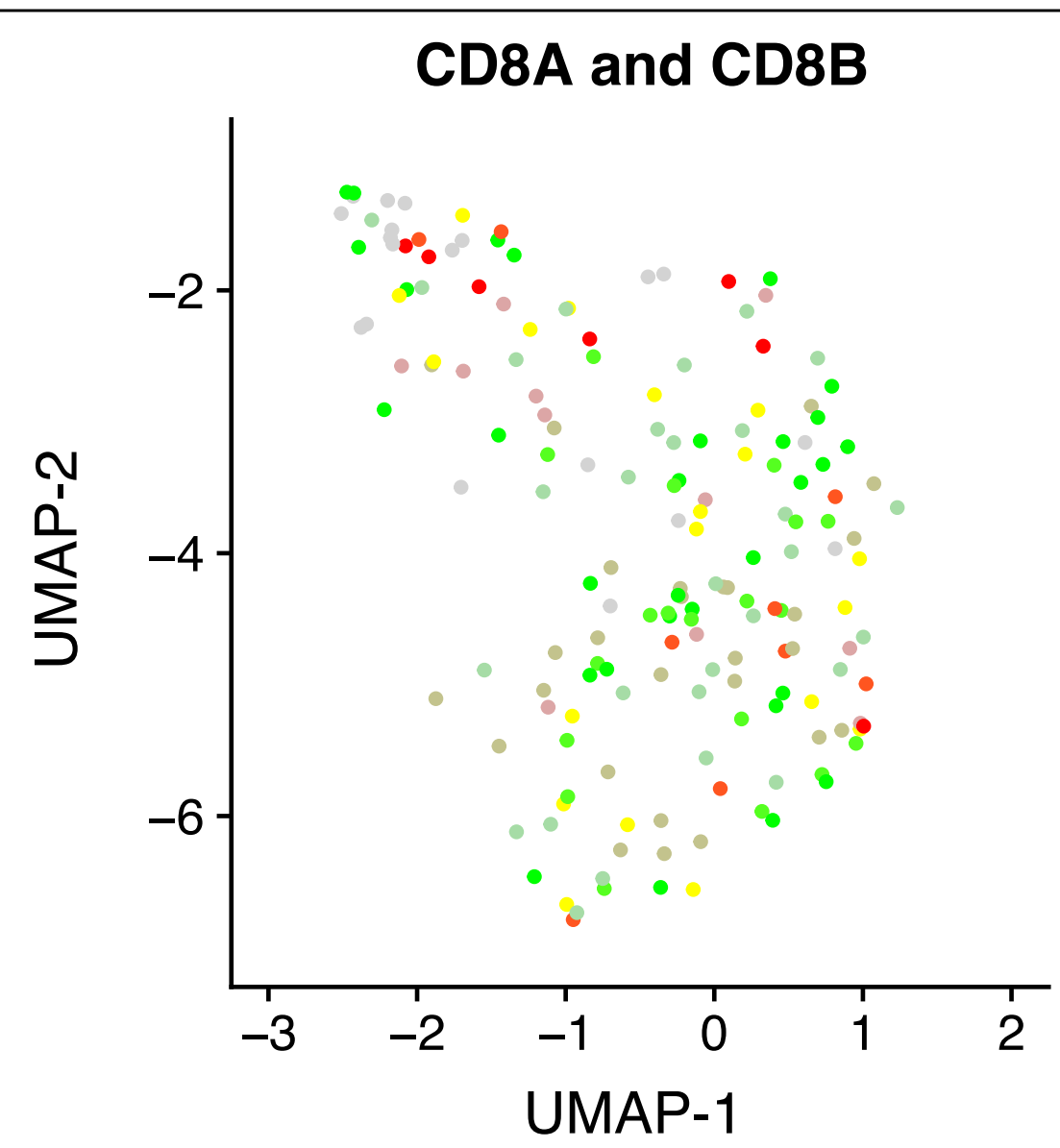

Expression Level

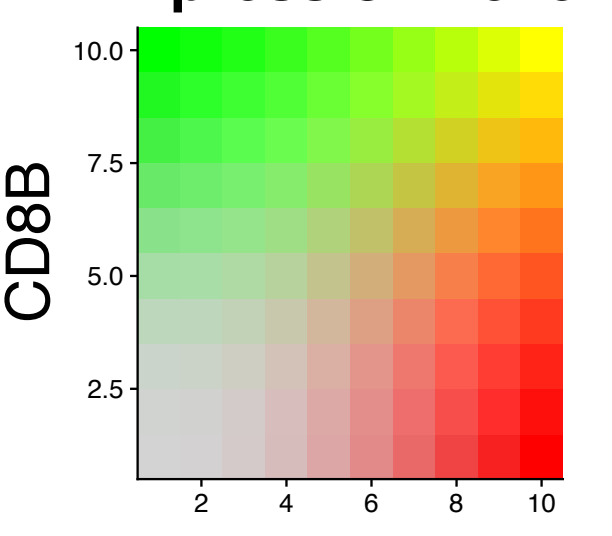

CD8A
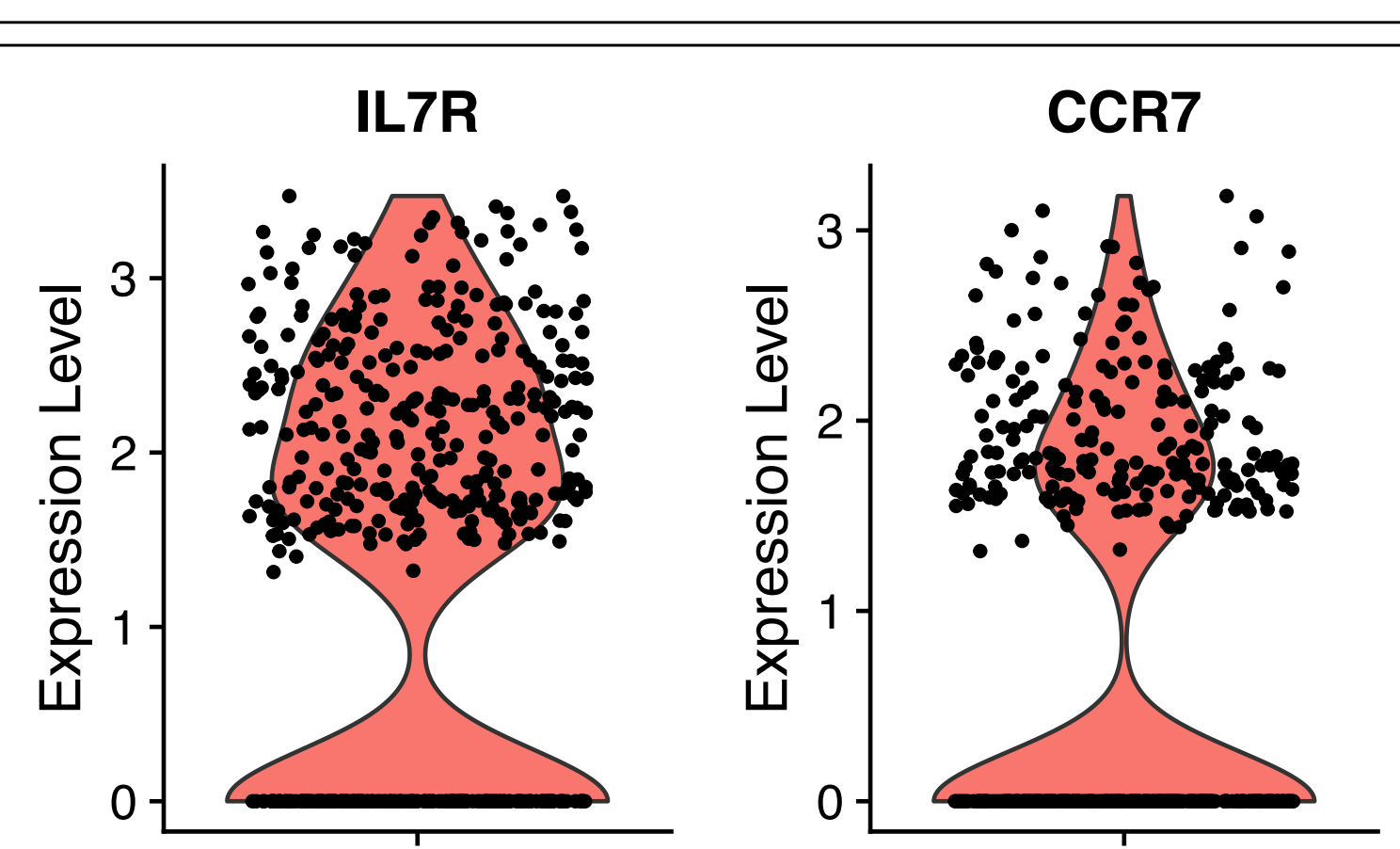

Seurat: CD4 T Naive

HieRFIT: CD4 T Treg, CD4 T cells, CD4 T Naive, CD4 T Memory
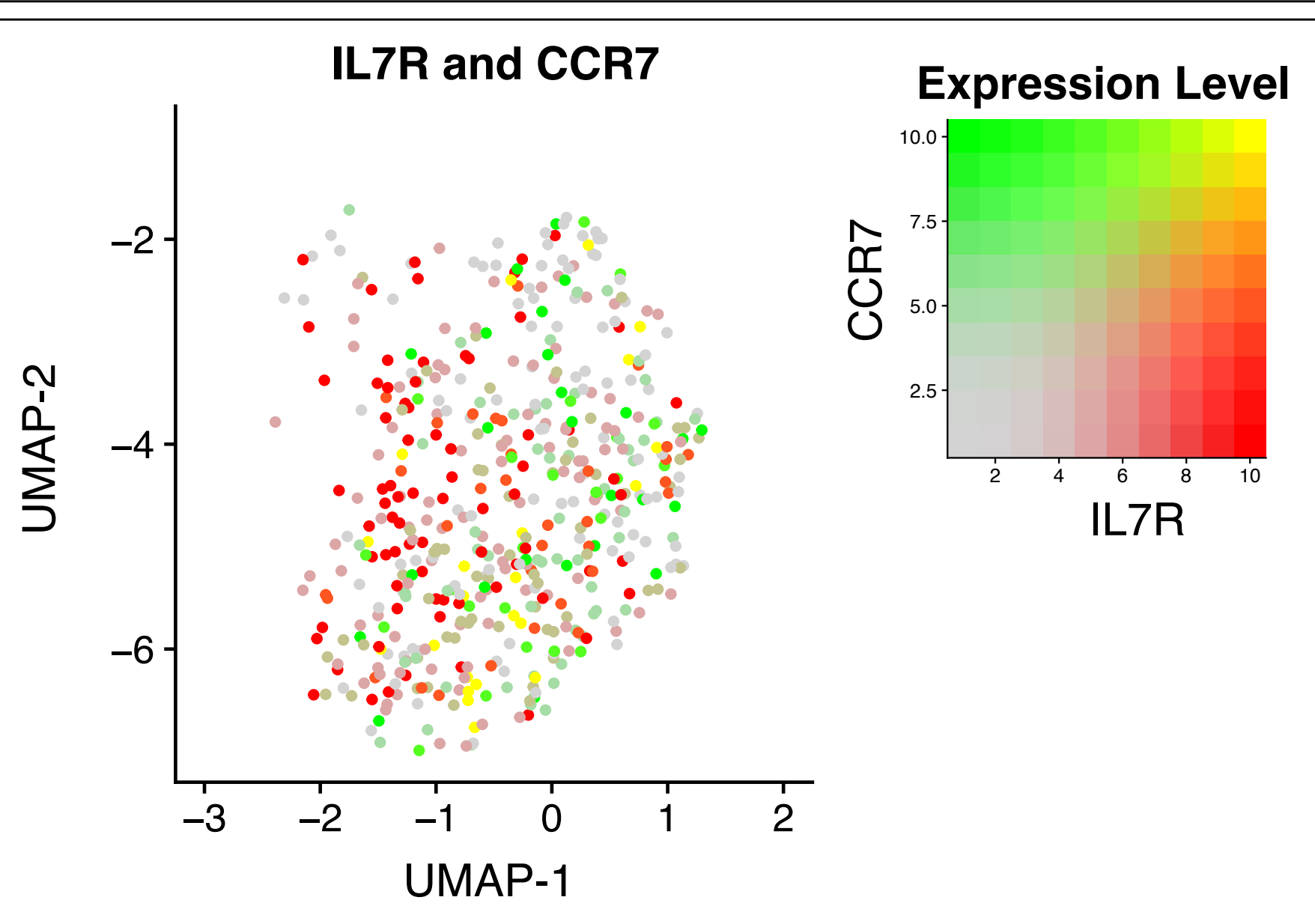

C

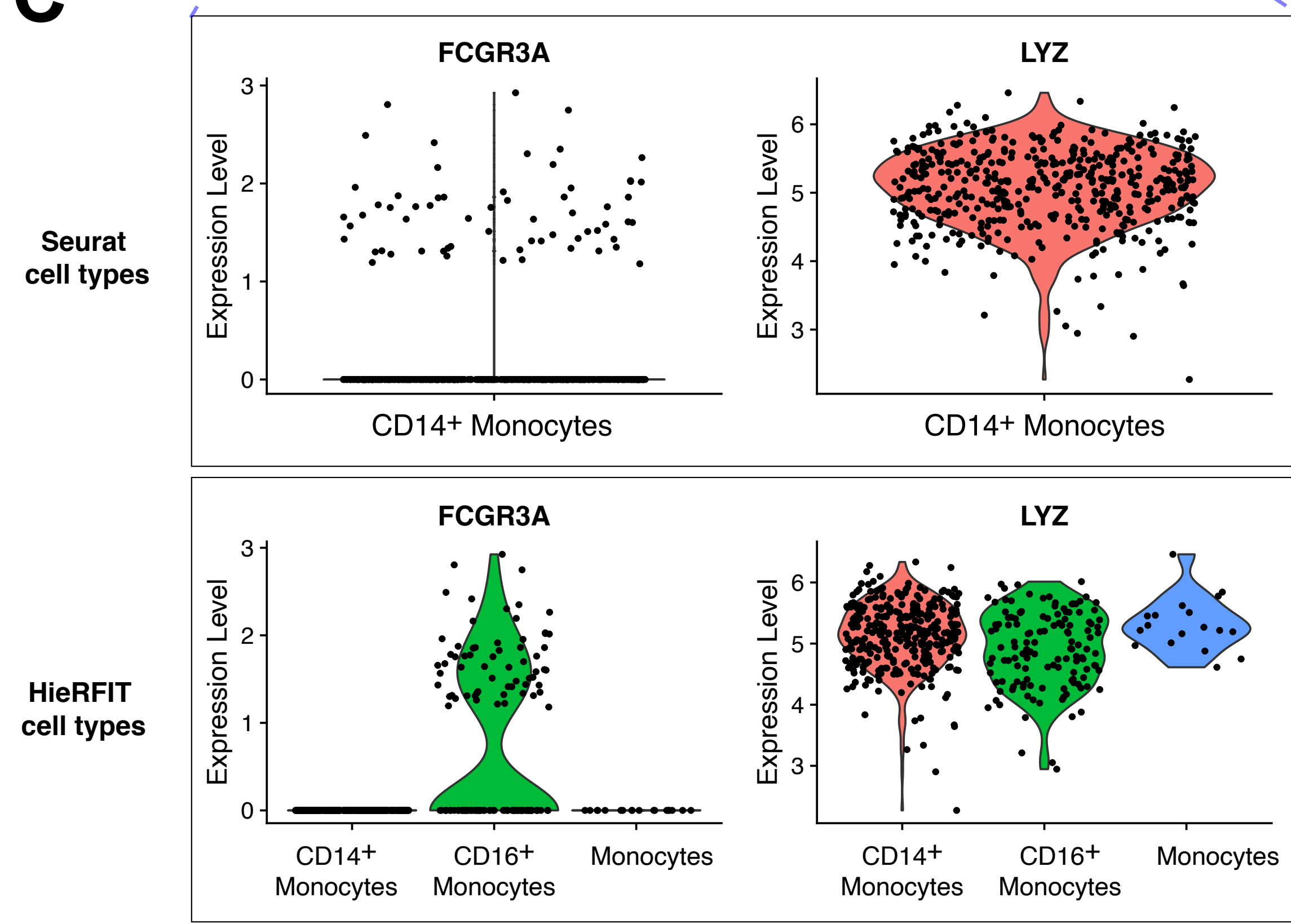


Figure 4

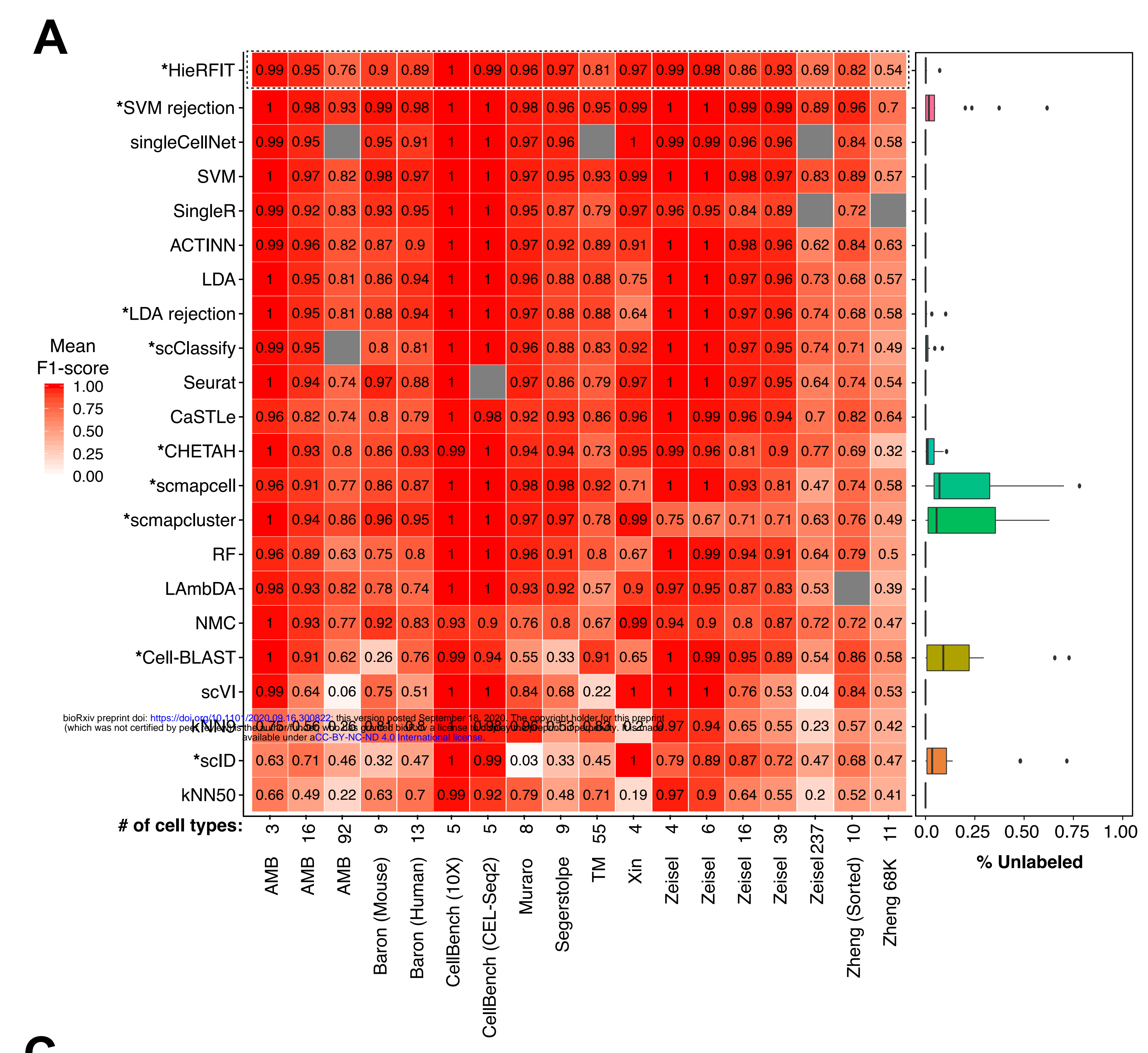

C

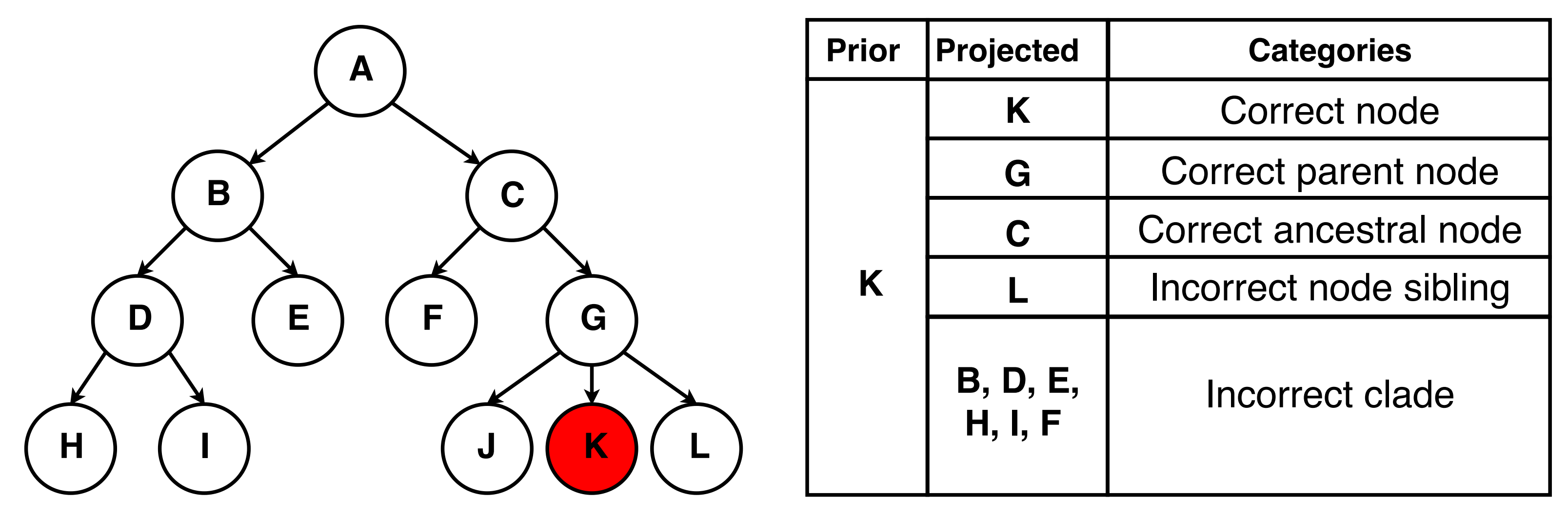

B

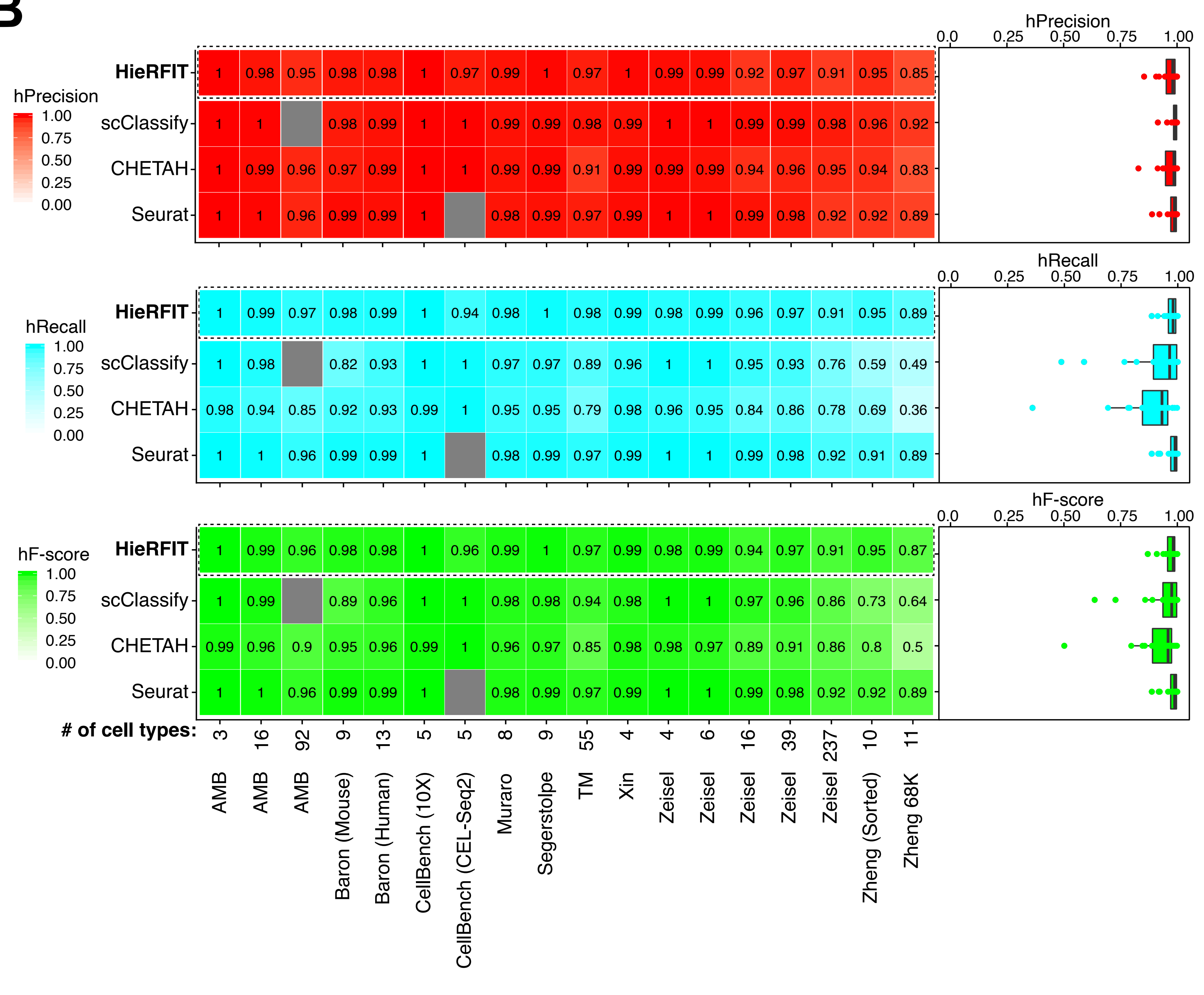

D

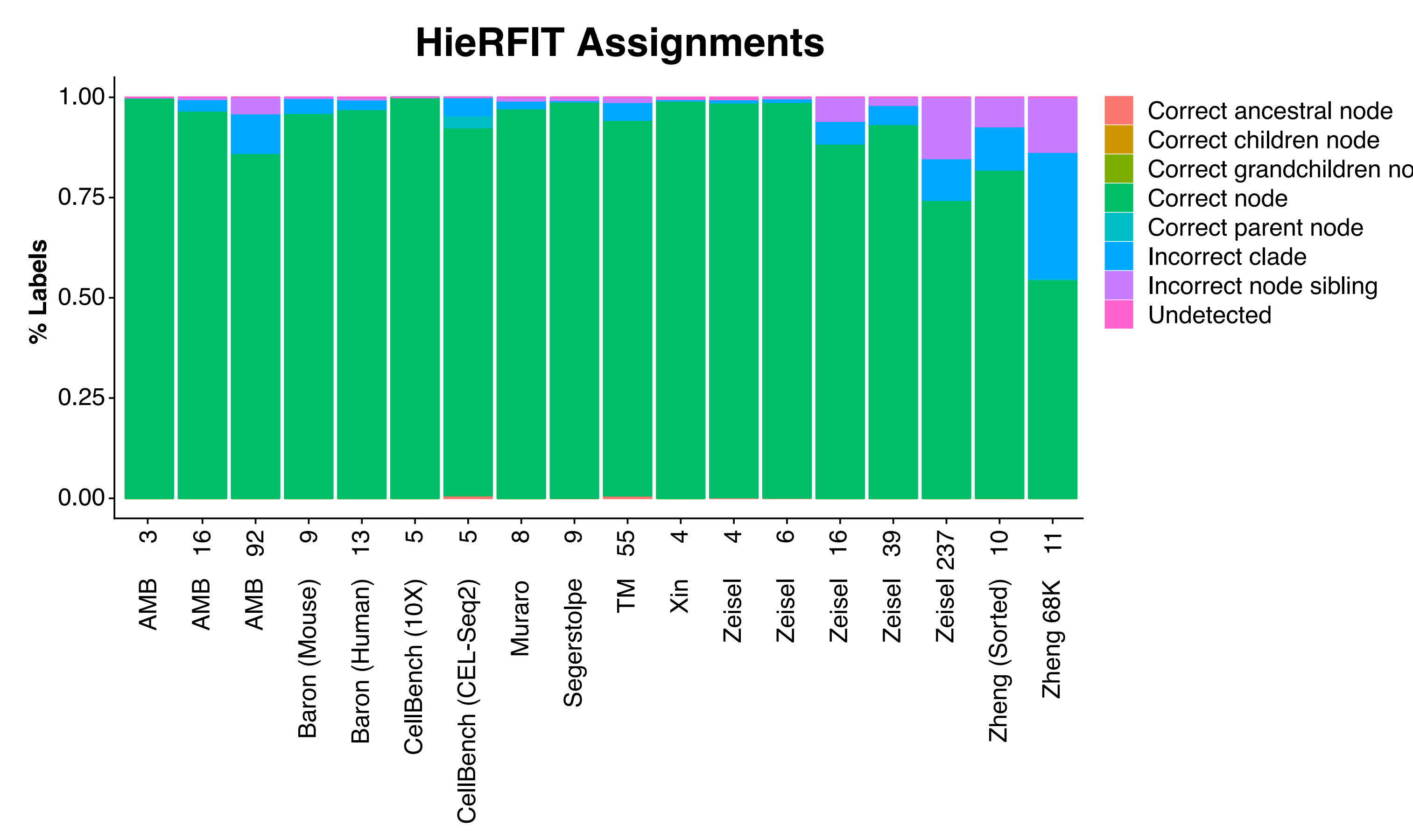




\section{Figure 5}

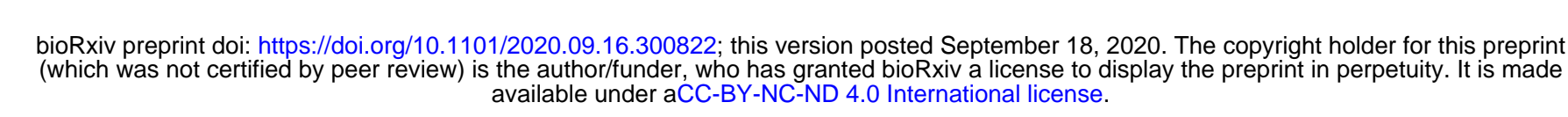

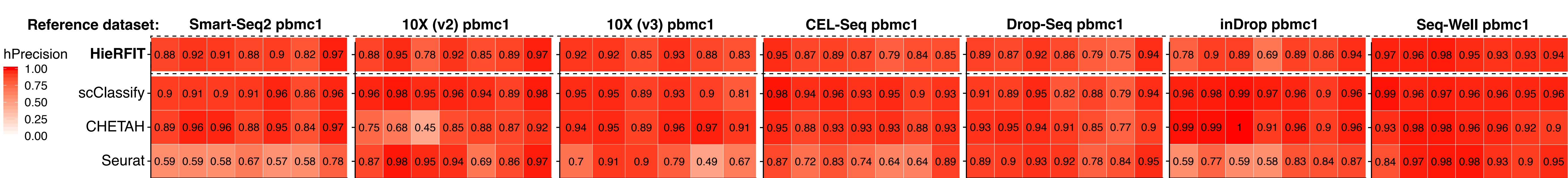

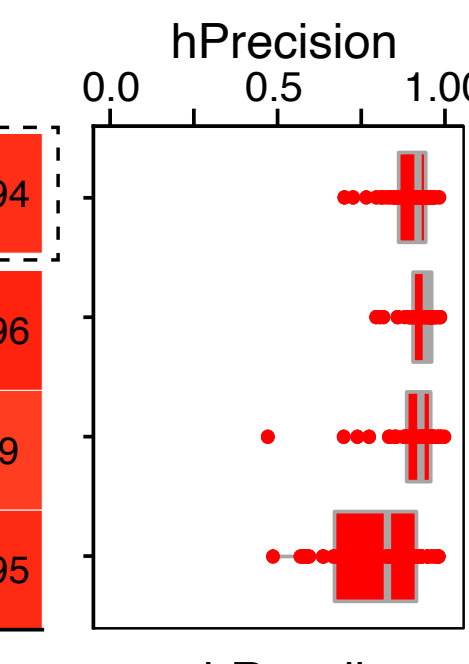

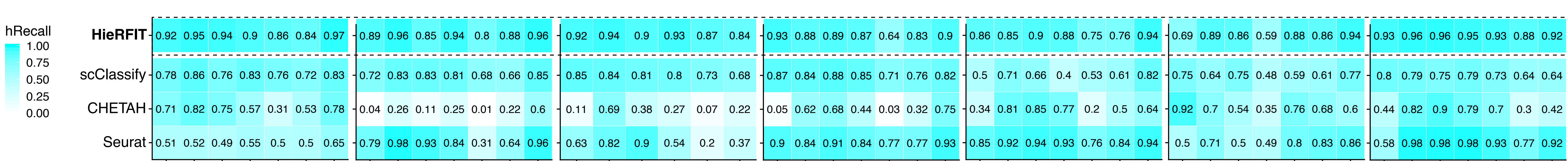

$\stackrel{\text { hRecall }}{0.5}, 1,00$

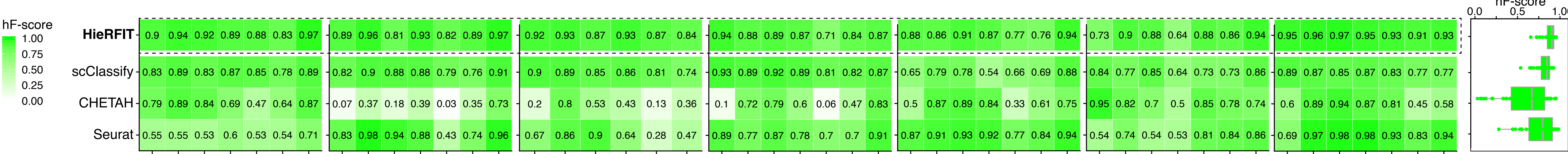
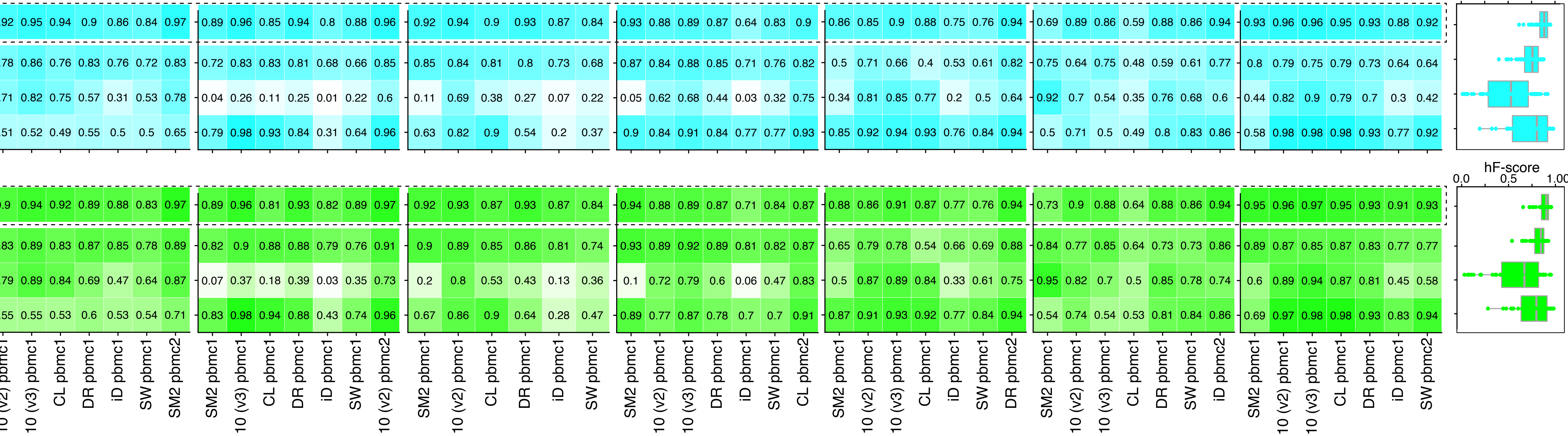